Noname manuscript No.

(will be inserted by the editor)

\title{
Outgassing History and Escape of the Martian Atmosphere and Water Inventory
}

\author{
Helmut Lammer • Eric Chassefière · Özgür \\ Karatekin - Achim Morschhauser - Paul \\ B. Niles · Olivier Mousis • Petra Odert . \\ Ute V. Möstl · Doris Breuer · Véronique \\ Dehant • Matthias Grott • Hannes Gröller . \\ Ernst Hauber • Lê Binh San Pham
}

Received: date / Accepted: date

\begin{abstract}
The evolution and escape of the martian atmosphere and the planet's water inventory can be separated into an early and late evolutionary epoch. The first epoch started from the planet's origin and lasted $~ 500$ Myr. Because of the high EUV flux of the young Sun and Mars' low gravity it was accompanied by hydrodynamic blow-off of hydrogen and strong thermal escape rates of dragged heavier species such as $\mathrm{O}$ and $\mathrm{C}$ atoms. After the main part of the protoatmosphere was lost, impact-related volatiles and mantle outgassing may have resulted in accumulation of a secondary $\mathrm{CO}_{2}$ atmosphere of a few tens to a few hundred mbar around $\sim 4-4.3$ Gyr ago. The evolution of the atmospheric surface pressure and
\end{abstract}

H. Lammer, H. Gröller, P. Odert

Space Research Institute, Austrian Academy of Sciences, Schmiedlstr. 6, A-8042 Graz, Austria

E-mail: helmut.lammer@oeaw.ac.at

E. Chassefière

Univ. Paris-Sud, Laboratoire IDES, CNRS, UMR8148, Orsay, F-91405, France

V. Dehant, Ö. Karatekin, L.B.S. Pham

Royal Observatory of Belgium, Brussels, Belgium

D. Breuer, M. Grott, E. Hauber, A. Morschhauser

German Aerospace Center, Institute of Planetary Research, Rutherfordstr. 2, D-12489 Berlin, Germany

P.B. Niles

Astromaterials Research and Exploration Science Johnson Space Center, NASA, Houston Texas, USA

\section{O. Mousis}

Observatoire de Besançon, 41 bis, avenue de l'Observatoire B.P. 1615 avenue de l'Observatoire, F-25010 Besançon, France,

and Université de Toulouse; UPS-OMP; CNRS-INSU; IRAP; 14 Avenue Edouard Belin, 31400 Toulouse, France 
water inventory of such a secondary atmosphere during the second epoch which lasted from the end of the Noachian until today was most likely determined by a complex interplay of various nonthermal atmospheric escape processes, impacts, carbonate precipitation, and serpentinization during the Hesperian and Amazonian epochs which led to the present day surface pressure.

Keywords Early Mars, young Sun, magma ocean, volcanic outgassing, impacts, thermal escape, nonthermal escape, atmospheric evolution

\section{Introduction}

The present martian atmosphere is the result of numerous interacting processes. On the one hand, these include atmospheric sinks such as erosion by impacts, thermal and nonthermal escape, extreme ultraviolet (EUV) radiation, as well as solar wind forcing (e.g. Lundin et al. 2007). On the other hand, atmospheric sources such as volcanic outgassing or delivery of volatiles by impacts have also to be taken into account for understanding atmospheric evolution. Furthermore, the atmosphere can interact with crustal reservoirs by $\mathrm{CO}_{2}$ weathering and hydration processes, which occur at the surface and/or in the crust (e.g., Zent and Quinn 1995; Bandfield et al. 2003; Becker et al. 2003; Lundin et al. 2007; Lammer et al. 2008; Pham et al. 2009; Tian et al. 2009; Philips et al. 2010). A schematic synopsis of these interactions is presented in Fig. 1.

The aim of this work is to review the latest knowledge on the evolution of the martian atmosphere since the planet's origin $\sim 4.55$ Gyr ago. In Sect. 2 we discuss the delivery of volatiles, the planet's early hydrogen-rich protoatmosphere, and point out possible reasons why there is an apparent deficiency of noble gases in the present atmosphere. In Sect. 3 we discuss different approaches to constrain volcanic outgassing rates of $\mathrm{CO}_{2}$ and $\mathrm{H}_{2} \mathrm{O}$. In Sect. 4 we consider the role of atmospheric impact erosion and delivery in the early martian environment. In Sect. 5, we discuss the efficiency of EUV-powered escape during the early Noachian and its influence on the growth of a secondary $\mathrm{CO}_{2}$ atmosphere. In Sect. 6 we briefly address consequences of the late heavy bombardment (LHB) on the martian atmosphere and its climate, $23.7-4$ Gyr ago. Finally, Sects. 7 and 8 focus on nonthermal atmospheric escape to space and on possible surface sinks of $\mathrm{CO}_{2}$ and $\mathrm{H}_{2} \mathrm{O}$ allowing the surface pressure to reach its present-day value.

\section{Origin and Delivery of Volatiles to Mars}

The sources and evolutionary histories of volatiles composing the martian atmosphere are poorly understood. They are related to the sources that delivered significant amounts of water to early Mars, which have implications for the formation of the planet's protoatmosphere. Furthermore, isotope variations in volatiles have the potential to provide insights into the origin and atmosphere modification processes in terrestrial planets, possibly related to the observation that the noble gases appear strongly depleted in the martian atmosphere compared to those of Earth and Venus. 


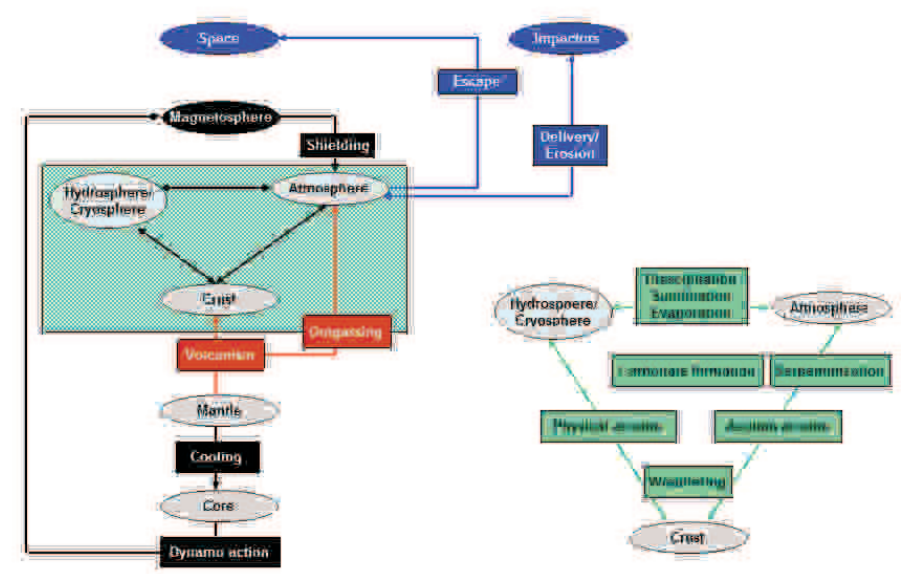

Fig. 1 Sketch showing important interactions between the main reservoirs, i.e. atmosphere, hydrosphere/cryosphere, crust, mantle and core (ovals in light blue), that have been addressed in the present work. Volcanism results in the formation of the crust and the associated degassing of the mantle produces an atmosphere with time (red boxes). Dynamo action in the core, which is triggered by efficient heat transfer in the mantle, and the subsequent shielding of the atmosphere prevents or reduces atmospheric erosion by non-thermal processes (black boxes). The erosion of the atmosphere to space can be caused by solar influx or by impacts (blue boxes). The latter may also deliver volatiles to the atmosphere depending on the impactors size and composition. The complex interactions between atmosphere, hydrosphere/cryosphere and crust (green dotted area) are shown in more detail on the right side (green boxes).

\subsection{Water Delivery and Formation of the Martian Protoatmosphere}

Four main processes are responsible for the early formation of an atmosphere:

- capture and accumulation of gasses from the planetary nebula,

- catastrophic outgassing due to magma ocean solidification,

- impacts,

and

- later degassing by volcanic processes.

As long as nebula gas is present, growing protoplanets can capture hydrogen and He which form gaseous envelopes around the rocky core (e.g., Hayashi et al. 1979; Rafikov 2006). Depending on the host star's radiation and plasma outflow, the nebula dissipation time, the planet's orbital location and the number and orbital location of additional planets in the system, according to Hayashi et al. (1979), planetary embryos with the mass of $\sim 0.1 M_{\text {Earth }}$ can capture hydrogen and other nebula gas from the nebula during $\sim 3$ Myr with an equivalent amount of up to $\sim 55$ times the hydrogen which is, present in the Earth's present day ocean. Furthermore, noble gases delivered by comets accreted during this period were mixed with volatiles remaining after an episode of strong atmospheric escape. 
The initial water inventory of a planet is acquired from colliding planetesimals, growing planetary embryos, impacting asteroids and comets (e.g., Lunine et al. 2003; Brasser 2012). Lunine et al. (2003) estimated the cumulative collision probability between small bodies and Mars and found that Mars' initial water inventory may have equivalent to $~ 0.06-0.27$ times that of an Earth ocean (EO), corresponding to a martian surface pressure of $\sim 10-100$ bar. Other simulations which considered different impact regimes suggest that Mars could also have been drier (Horner 2009). In a more recent study, Walsh et al. (2011) argues that the small mass of Mars indicates that the terrestrial planets in the Solar System have formed from a narrow material annulus, rather than a disc extending to Jupiter. In such a scenario the truncation of the outer edge of the disc was the result of the migration of the gas giants, which kept the martian mass small. From cosmochemical constraints one can argue that Mars formed in a couple of Myr and can be considered in agreement with the latest dynamical models as a planetary embryo that never grew to a real planet. In such a case most of Mars' materials consisted of building blocks that formed in a region at $\sim 2-3 \mathrm{AU}$, and therefore, were more $\mathrm{H}_{2} \mathrm{O}$-rich compared to the materials which formed Earth and Venus. From these arguments Brasser (2012) suggests that Mars may have consisted of $\sim 0.1-0.2$ wt. $\%$ of water.

A substantial part of the initial inventory of volatiles could have been outgassed as a consequence of the solidifcation of an early magma ocean (Elkins-Tanton 2008). Water and carbon dioxide enter solidifying minerals in only small quantities and are enriched in magma ocean liquids as solidification proceeds. Close to the surface at low pressure these volatiles degas into the growing atmosphere. Depending on the initial water/volatile content, which was built-in the planetary body during its growth and the depth of the possible magma ocean $z_{\text {mag }}$, steam atmospheres with a surface pressure between $\sim 30\left(0.05\right.$ wt. $\% \mathrm{H}_{2} \mathrm{O}, 0.01$ wt. $\% \mathrm{CO}_{2}$, $\left.z_{\mathrm{mag}} \sim 500 \mathrm{~km}\right)$ to $\sim 800$ bar $\left(0.5 \mathrm{wt} . \% \mathrm{H}_{2} \mathrm{O}, 0.1\right.$ wt. $\% \mathrm{CO}_{2}, z_{\mathrm{mag}} \sim 2000 \mathrm{~km}$ ) (see Table 3, Elkins-Tanton 2008) could have been catastrophically outgassed. If early Mars consisted of $\sim 0.1-0.2$ wt.\% water (Brasser 2012) then a steam atmosphere with a surface pressure of more than $\sim 60$ bar could have been catastrophically outgassed (Elkins-Tanton 2008). Although it is assumend that most volatiles are degassed into the early atmosphere, a geodynamically significant quantity is still sequestered in the solid cumulates. The amount is estimated to be as much as $750 \mathrm{ppm}$ by weight $\mathrm{OH}$ for an initial water content of $0.5 \mathrm{wt} . \%$, and a minimum of $10 \mathrm{ppm}$ by weight in the driest cumulates of models beginning with just 0.05 wt.\% water (Elkins-Tanton 2008). Even more water in the martian interior can be expected after the magma ocean solidification phase in the case of a shallow magma ocean in particular in the deep unmolden primordial mantle. In any case, these small water contents significantly lower the viscosity and possibly the melting temperature of mantle materials, facilitating later volcanism, as discussed below.

The early steam atmosphere could have remained stable for a few tens of Myr. During this early stage, environmental conditions were determined by a high surface temperature and frequent impacts, which could have reached up to $\sim 1500$ $\mathrm{K}$ due to thermal blanketing and frequent impacts (e.g., Matsui and Abe 1986). If such a steam atmosphere is not lost upon cooling, the remaining $\mathrm{H}_{2} \mathrm{O}$ vapor can condense and produces liquid water on the surface or ice in case of a cold climate (e.g., Chassefière 1996). 
If Mars originated with $\sim 0.1-0.2 \mathrm{wt} \% \mathrm{H}_{2} \mathrm{O}$, as long as the planet was surrounded by a captured dense nebula-based hydrogen envelope, magma ocean related outgassed greenhouse gases $\left(\mathrm{H}_{2} \mathrm{O}, \mathrm{CO}_{2}, \mathrm{CH}_{4}, \mathrm{NH}_{3}\right)$ would have been protected against dissociation because these heavy molecules would remain closer to the planet's surface compared to the lighter hydrogen in the upper atmosphere. Depending on the amount and the lifetime of accumulated nebula gas and its evaporation time, a combination of a possible $\mathrm{H}_{2}$ greenhouse (Pierrehumbert and Gaidos 2011; Wordsworth 2012) and the outgassed greenhouse gases may have provided warm and wet conditions on the martian surface for a few tens of Myr.

Finally, it is important to note that the previous investigations of planetary formation suffer from several unknowns including the sources of impactors across the inner Solar System. Such work would require far more detailed model populations for the cometary and asteroidal sources, and would have to include a study of the effects of Oort cloud comets. Because the results of Lunine et al. (2003) and Brasser (2012) are different from those of Horner et al. (2009), it is obvious that our knowledge of terrestrial planet formation and hydration is currently insufficient because it is not possible to predict the real initial deuteration level on each of the planets considered. This piece of evidence, combined with the fact that the $\mathrm{D} / \mathrm{H}$ ratio in $\mathrm{H}_{2} \mathrm{O}$ in comets is not homogeneous (Hartogh et al. 2011), indicate that the water delivery mechanisms to the terrestrial planets can only be established within an uncertainty range.

\subsection{The Apparent Noble Gas Deficiency of the Martian Atmosphere}

The difference between the measured atmospheric abundances of non-radiogenic noble gases in Venus, Earth, and Mars is striking. It is well known that these abundances decline dramatically as one moves outward from Venus to Mars within the inner Solar System, with these two planets differing in abundance by up to two orders of magnitude (see Fig. 2). Therefore, understanding this variation is a key issue in understanding how the initial atmospheres of the terrestrial planets evolved to their current composition, and requires to study the different delivery mechanisms of the volatiles accreted by these planets (Pepin 1991; 2006; Owen et al. 1992; Owen and Bar-Nun 1995; Dauphas 2003; Marty and Meibom 2007).

In this context, recent $n$-body simulations have been performed by Horner et al. (2009) in order to study the impact rates experienced by the terrestrial planets as a result of diverse populations of potential impactors. These authors considered a wide range of plausible planetary formation scenarios for the terrestrial planets, and found that the different impact regimes experienced by Venus, Earth, and Mars could have resulted in significant differences between their individual hydration states over the course of their formation and evolution. Horner et al. (2009) found that, on average, the Earth most likely received a flux of impacting comets which is 3.4 times higher than that experienced by Mars. Assuming that the mass of noble gases delivered by comets to the terrestrial planets is proportional to the rate at which they impacted upon them, it is possible to derive $X_{\mathrm{E}} / X_{\mathrm{M}}$ (the ratio of the noble gas abundances (as a fraction of the total mass of the planet) between Earth and Mars) from the ratio of the number of comets impacting upon those 


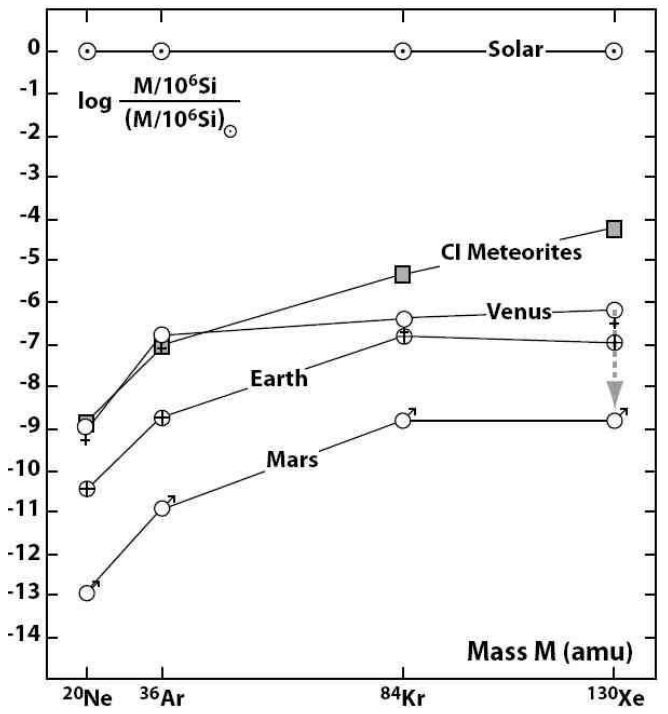

Fig. 2 Measured abundances of $\mathrm{Ne}, \mathrm{Ar}, \mathrm{Kr}$, and $\mathrm{Xe}$ in the atmospheres of the terrestrial planets and primitive CI meteorites. The values shown for these gases are presented relative to their solar abundances, in units of atoms per $10^{6} \mathrm{Si}$ atoms (adapted from Fig. 2 of Pepin 1991). The vertical arrow pointing down indicates that the Venus atmospheric abundance of Xe is only an upper limit.

two planets $N_{E} / N_{M}(\sim 3.4)$, through the following relation (Mousis et al. 2010)

$$
\frac{X_{\mathrm{E}}}{X_{\mathrm{M}}}=\frac{N_{\mathrm{E}}}{N_{\mathrm{M}}} \frac{M_{\mathrm{M}}}{M_{\mathrm{E}}}
$$

where $M_{\mathrm{E}}$ and $M_{\mathrm{M}}$ are the masses of Earth and Mars, respectively. From this relation, one can infer that the average noble gas abundance on Earth should be $\sim 0.37$ times the martian noble gas abundances if these volatiles were solely delivered by comets. This result differs significantly from that inferred from measurements of noble gas abundances, which are observed to be approximately two orders of magnitude larger for the Earth compared to Mars. As a result, subsequent processes that occurred preferably during the post-impact period of Mars are required in order to explain its present-day atmospheric composition. It has been proposed that atmospheric escape could have strongly altered the composition of the atmospheres of terrestrial planets (Pepin 1991; 1997; Dauphas 2003; Jakosky et al. 1994; Chassefière and Leblanc 2004). This hypothesis is supported by both Mars (SNC meteorites) and Earth, which show substantial fractionation of Xe isotopes compared to the plausible primitive sources of noble gases, i.e., solar wind (SW-Xe), meteorites $(\mathrm{Q}-\mathrm{Xe})$, or the hypothetical U-Xe source (Pepin 2006). This fractionation then suggests important losses of Xe and other noble gases from the early atmospheres of the Earth and Mars. Impact related loss processes might have been more important for the Earth and Mars than Venus because the latter planet would have escaped impacts of the magnitude that formed the Moon (Canup and Asphaug 2001) or created the largest basins on Mars (Andrews-Hanna et al. 2008). In the case of the Earth, the noble gas fractionation episode could have also been 
driven by impacts (Pepin 1991; Pepin 1997; Dauphas 2003) in combination with the high EUV radiation of the young Sun (e.g., Ribas es al. 2005; Lammer et al. 2008).

Thus, in the case of Mars, the combination of impacts, EUV-powered hydrodynamic escape, planetary degassing, and fractionation by nonthermal atmospheric escape processes (Jakosky et al. 1994; Luhmann et al. 1992; Carr 1999; Chassefière and Leblanc 2004; Lammer et al. 2008) might have played an important role in sculpting the pattern of the noble gas abundances observed today. An alternative hypothesis proposed to explain the $\mathrm{Kr}$ and Xe abundance differences between Earth and Mars is the presence of large amounts of $\mathrm{CO}_{2}$-dominated clathrates in the martian soil that would have efficiently sequestered these noble gases (Mousis et al. 2010; Mousis et al. 2012). In this scenario, these noble gases would have been trapped in clathrates $\sim 4$ Gyr b.p. when the $\mathrm{CO}_{2}$ surface pressure was expected to be of the order of a few tens to a few hundred mbar (Mousis et al. 2012). This scenario implies that the ${ }^{36} \mathrm{Ar},{ }^{84} \mathrm{Kr}$, and ${ }^{130} \mathrm{Xe}$ abundances measured in the planet's atmosphere are not representative of its global noble gas budget. Depending on the amount of existing clathrates, the volume of noble gases trapped in these crystalline structures could be much larger than those measured in the atmosphere. In this context, two different scenarios have been proposed by Mousis et al. (2010) to explain the differences between the $\mathrm{Ne}$ and Ar abundances of the terrestrial planets.

In the first scenario, cometary bombardment of the planets would have occurred at epochs contemporary with the existence of their primary atmospheres. Comets would have been the carriers of Ar, Kr, and Xe, while Ne would have been gravitationally captured by the terrestrial planets (Owen et al. 1992). Only $\mathrm{Ne}$ and Ar would have been fractionated due to thermal and nonthermal atmospheric escape, while the abundances of the heavier noble gases would have been poorly affected by such losses. In this scenario, the combination of processes, such as escape of $\mathrm{Ne}$ and $\mathrm{Ar}$, cometary bombardment at the epochs of existence of primary planetary atmospheres, and the sequestration of krypton and xenon in the martian clathrates, would then explain the observed noble gas abundance differences between the Earth and Mars. However, this scenario leads to an important chronological issue because depending on a the hydrogen/He amount of the captured nebula-based protoatmosphere it existed most likely only during the first few to several tens of Myr (Halliday 2003; Pepin 2006; Lammer et al. 2012).

On the other hand one should also note that heavy noble gases could have been supplied during the LHB (Marty and Meibom 2007). In such a second scenario, Mousis et al. (2010) considered impacting comets that contained significantly smaller amounts of Ar, an idea supported by predictions of noble gas abundances in these bodies, provided that they are formed from clathrates in the solar nebula (Iro et al. 2003). Here, Ne and Ar would have been supplied to the terrestrial planets via the gravitational capture of their primary atmospheres and comets would have been the carriers of $\mathrm{Kr}$ and Xe only. In this case, the cometary bombardment of the terrestrial planets could have occurred after the formation of their protoatmospheres because only the neon and argon abundances observed today would have been engendered by the escape-fractionation processes in these atmospheres.

Both scenarios preclude the possibility that material with a CI chondrite-like composition could be the main source of noble gases in terrestrial planets because 
the trend described by the chondritic noble gas abundances as a function of their atomic mass does not reflect those observed on Venus, the Earth, and Mars (Pepin et al. 1992; Owen and Bar-Nun 1995). If the composition is similar to that of CI chondrites, this then excludes the hypothesis of noble gas outgassing from the interior of Mars and also the scenario of asteroidal bombardment. Irrespective of the scenario envisaged, this work does not preclude the possibility that a fraction of the heavy noble gases could have been captured by the Earth and Mars during the acquisition of nebula-based protoatmospheres. In the first scenario, the fraction of $\mathrm{Kr}$ and Xe accreted in this way should be low compared to the amount supplied by comets since these noble gases are not expected to have been strongly fractionated by atmospheric escape. In the second scenario, the fraction of Kr and Xe captured gravitationally by the terrestrial planets could be large if escape was efficient.

\section{Outgassing and Growth of a Secondary Atmosphere}

3.1 Estimation of the martian water-ice reservoir by the atmospheric $\mathrm{D} / \mathrm{H}$ ratio

Volcanic outgassing is one of the main sources of volatiles for the Martian atmosphere and provides an important link between mantle and atmospheric geochemical reservoirs. Information on exchange processes between the different reservoirs is contained in the atmospheric isotopic ratios $R$ of elements such as hydrogen, carbon, and the noble gases. $R$ may change as a function of time as lighter isotopes can escape to space more efficiently than their heavier counterparts. Overall, the efficiency of isotopic fractionation depends on the size $S$ of the considered reservoir, the total escape flux $\phi$, and the relative efficiency of isotopic escape which may be expressed by the fractionation factor $f$ (Donahue 2004).

The ratio of the sizes of the past and present reservoirs in isotopic equilibrium with the atmosphere can be calculated if the respective isotopic ratios are known and is given by Donahue (1995)

$$
\frac{S_{\mathrm{t}}}{S_{\mathrm{p}}}=\left(\frac{R_{\mathrm{p}}}{R_{\mathrm{t}}}\right)^{1 /(1-f)}
$$

where variables with index $p$ and $t$ refer to the present and past values, respectively. If an initial isotopic ratio is assumed for $R_{\mathrm{t}}$, the size of the reservoir when it has last been reset to this value will be obtained. This reset may have happened due to strong volcanic outgassing, delivery of additional material by impacts, or a sudden exchange with other reservoirs not in isotopic equilibrium with the atmosphere. In this way, isotopic ratios found in Martian meteorites can be used for $R_{\mathrm{t}}$ to obtain the size of the reservoir at the time of their crystallization.

In the following, we will consider the size of the water reservoir and use the isotopic ratio of deuterium (D) and atomic hydrogen $(\mathrm{H})\left(D_{0} / H_{0}\right)$. A compilation of different $\mathrm{D} / \mathrm{H}$ isotopic ratios in the martian atmosphere, terrestrial sea water as well as comets and martian meteorites is given in Table 1 . The initial D/H ratio was modified from its initial ratio to the present one by atmospheric escape processes. One way to estimate the initial ratio on Mars is to assume that isotopic ratios on Earth and Mars were identical following accretion. Given that the amount of water present in Earth's oceans is very large, isotopic ratios have probably changed by less than $0.2 \%$ since accretion (Donahue 2001) and $D_{0} / H_{0}$ can be approximated 
$\mathrm{D} / \mathrm{H}$ ratio in the martian atmosphere, the terrestrial sea water, comets and various martian meteorites. The crystallization ages and ejection ages of the meteorites are taken from Nyquist et al. (2001) and for ALH 84001 from Turner et al. (1997). The measured D/H values are taken from Leshin et al. (1996) and are also given in units of terrestrial sea water D/H. The ejection age refers to the estimated time of ejection from the Martian surface.

\begin{tabular}{l|cccc}
\hline Reservoir & $\mathrm{D} / \mathrm{H}\left[1 \times 10^{-4}\right]$ & $\mathrm{D} / \mathrm{H}[\mathrm{SMOW}]$ & Cryst. age $[\mathrm{Myr}]$ & Ejection age $[\mathrm{Myr}]$ \\
\hline Mars atmosphere & 8.0 & 5.13 & \\
Terrestrial sea water (SMOW) & 1.56 & $\sim 2.05$ & 1.00 \\
Comets & $\sim 3.2$ & & & \\
\hline martian meteorites & & 1.57 & $3920 \pm 40$ & $15.0 \pm 0.8$ \\
\hline AH 84001 & 2.45 & $0.96-1.03$ & $1340 \pm 50$ & $11.3 \pm 0.6$ \\
Chassigny & $1.49-1.6$ & $1.44-1.75$ & $1270 \pm 50$ & $10.75 \pm 0.4$ \\
Nakhla & $2.24-2.73$ & $1.58-1.79$ & $1320 \pm 20$ & $11.9 \pm 2.2$ \\
Lafayette & $2.47-2.8$ & 1.26 & $1330 \pm 10$ & $10.0 \pm 2.1$ \\
Governador Valadares & 1.97 & 2.10 & $177 \pm 3$ & $2.92 \pm 0.15$ \\
Zagami & 3.28 & 2.17 & $165 \pm 4$ & $2.73 \pm 0.2$ \\
Shergotty & 3.39 & 2.47 & $173 \pm 3$ & $0.73 \pm 0.15$ \\
Elephant Moraine 79001 & 3.86 & & & \\
\hline
\end{tabular}

by its present day value of the Standard Mean Ocean Water (SMOW). Another way to constrain $D_{0} / H_{0}$ is to calculate the Martian primordial composition from dynamical accretion models, which result in a primordial isotopic ratio $D_{0} / H_{0}$ between 1.2 and 1.6 times that of the SMOW (Lunine et al. 2003), indicating the range of uncertainty associated with this value. The current atmospheric $\mathrm{D} / \mathrm{H}$ ratio on Mars was measured with a $4 \mathrm{~m}$ reflecting telescope in combination with a Fourier transform spectrometer at Kitt Peak Observatory providing a significantly fractionated value of $R_{\mathrm{p}}=5.5 \pm 2$ SMOW (Krasnopolsky et al. 1997). From high resolution spectroscopic observations of $\mathrm{D}$ and $\mathrm{H}$ Lyman- $\alpha$ emissions of the martian hydrogen corona with the Hubble Space Telescope, the fractionation factor $f$ for $\mathrm{D}$ and $\mathrm{H}$ was estimated to be $\sim 0.016-0.02$ (Krasnopolsky et al. 1998; 2000). These values are significantly lower than the theoretically calculated value of 0.32 by Yung et al. (1988), resulting in larger reservoirs than previously assumed. Using $f=0.02$, the size $S_{0}$ of the water reservoir at the time when the isotopic ratio in the Martian atmosphere was last reset can be estimated from Eq. 2 and is shown in Fig. 3 as a function of the initial $\mathrm{D} / \mathrm{H}$ ratio with $D_{0} / H_{0}=1$ to 1.6 . Reservoir size is given in terms of its present-day size for three different values of the current $\mathrm{D} / \mathrm{H}$ ratio. This calculation implies that the past water reservoir was $2-8$ times larger than today, indicating that 50 to $88 \%$ of the past reservoir was lost.

In addition, the absolute size of the past water reservoir can be estimated from the total atmospheric escape flux (Donahue 2004). Results are sensitive to the assumed escape flux and early estimates arrived at a total past reservoir size corresponding to an equivalent global layer (EGL) of water between $0.2 \mathrm{~m}$ (Yung et al. 1988) and 30-80 m Jakosky et al. (1991). More recently, Krasnopolsky and Feldman (2001) estimated a total reservoir size of 65-120 m EGL, Lammer et al. (2003) obtained a value of 17-61 m EGL, while Donahue (2004) used isotopic ratios in the Zagami meteorite as an additional constraint to arrive at 100-800 m EGL. A scenario where serpentinization in the crust stored most of the ancient water reservoir has recently been proposed by Chassefière and Leblanc (2011c). They used the present D/H ratio to conclude that up to $\sim 400 \mathrm{~m}$ EGL of free water 


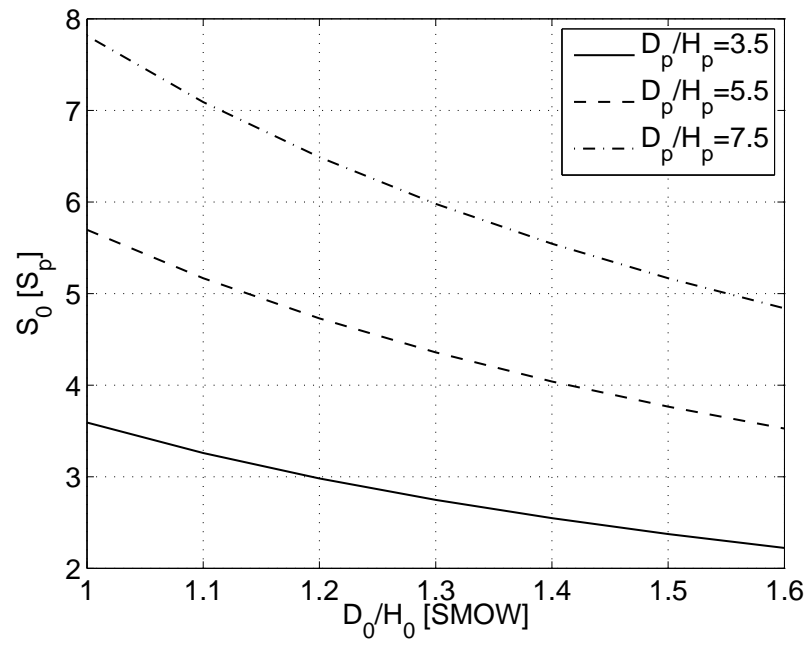

Fig. 3 Size of the martian water reservoir in isotopic equilibrium with the atmosphere as a function of the initial deuterium to hydrogen ratio $D_{0} / H_{0}$ for three different present-day isotopic ratios $D_{p} / H_{p}$. Reservoir size is given in terms of its present-day size $H_{p}$ and corresponds to the time when the isotopic ratio in the atmosphere was last reset to the initial ratio. The assumed fractionation factor $f$ is 0.02 , as measured with the Hubble Space Telescope (Krasnopolsky et al. 1998, 2000).

could hypothetically be stored in crustal serpentine, based on the assumption that $\mathrm{D}$ and $\mathrm{H}$ atoms released into the atmosphere during serpentinization have escaped and fractionated.

As the initial martian water inventory was most likely affected by hydrodynamic blow-off due to the young Sun's high EUV flux (cf. Sec. 5), H and D will have escaped unfractionated to space between $\sim 4.0-4.5$ Gyr ago. Therefore, these values may give only an estimate of the amount of volatiles delivered by impacts and volcanic outgassing until $\sim 4$ Gyr ago.

\subsection{Volcanic outgassing of $\mathrm{CO}_{2}$ and $\mathrm{H}_{2} \mathrm{O}$}

One way to quantify the rate of volcanic outgassing is to estimate the amount of crustal production as a function of time and to multiply this volume by the magma volatile content. In this case, the outgassing rate can be obtained from

$$
\frac{d M_{\mathrm{i}}^{\mathrm{atm}}}{d t} \propto \frac{d M_{\mathrm{cr}}}{d t} \eta X_{\mathrm{i}}^{\mathrm{melt}}
$$

where $M_{\mathrm{i}}^{\mathrm{atm}}$ is the outgassed mass of volatile species $i, M_{\mathrm{cr}}$ is the amount of extracted magma, $X_{\text {melt }}^{i}$ is the concentration of volatile species $i$ in the melt, and $\eta$ is an outgassing efficiency.

As the solubility of volatiles in magmas at surface pressure is low, essentially all dissolved volatiles will be released when erupting extrusively. For intrusive volcanism, it can be assumed that plutons do not contribute to volcanic outgassing 


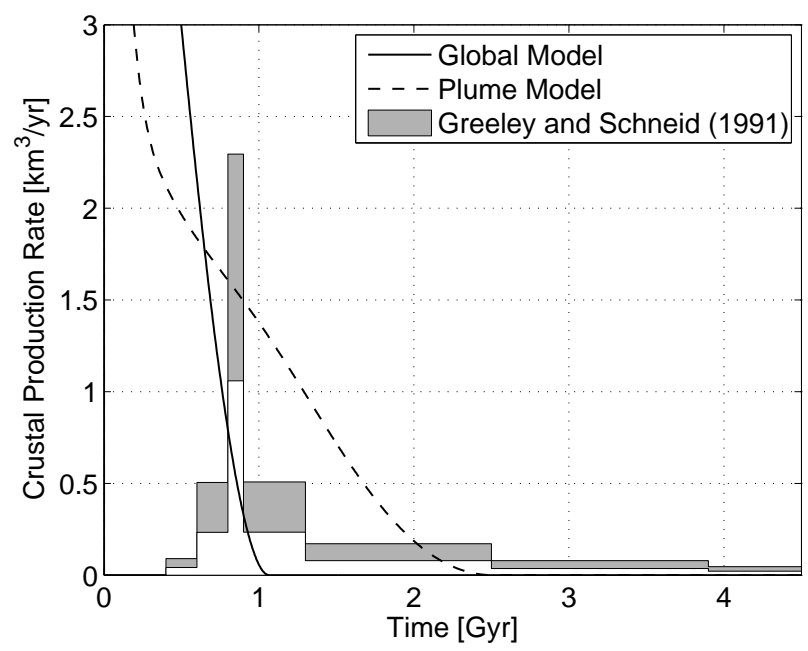

Fig. 4 Different estimates of crustal production rates as a function of time. The solid and dashed lines show the rates obtained with parameterized thermochemical evolution models assuming a global melt layer and melt generation in localized, hot mantle plumes, respectively (Grott et al. 2011; Morschhauser et al. 2011). The shaded area corresponds to the values obtained from photogeological estimates by Greeley and Schneid (1991) with ratios of extrusive to intrusive volcanism ranging from 1:5-1:12.

(O'Neill et al. 2007) and $R_{i}$ will depend on the ratio of intrusive to extrusive volcanism. However, as volatiles will be enriched in the remaining liqud during solidification, it is also possible that dissolved volatiles will outgas at depth and reach the atmosphere (Hirschmann et al. 1998). An intermediate approach is to assume that volatiles can be delivered to the surface as long as some crustal porosity is present at the depth of the intrusion (Grott et al. 2011). The volume of crustal production has been estimated from the photogeological record and crater counting has been used to age-date the corresponding surfaces. In this way, the rate of crustal production, i.e., the amount of crust produced as a function of time, has been determined. Lava volumes were obtained from the topology of partially filled impact craters by comparing their actual depth to the theoretical values obtained from depth-to-diameter scaling relations (Greeley and Schneid 1991). As only extrusions can be assessed, estimates of the ratio of extrusive to intrusive volcanism are necessary to obtain the total volume of produced crust. Also, older deposits may be covered by later extrusions and therefore early crust production rates may be underestimated.

Alternatively, numerical models of the thermochemical evolution of Mars can be used to calculate the globally averaged crustal production rates (Hauck and Phillips 2002; Breuer and Spohn 2006; Fraeman and Korenaga 2010; Morschhauser et al. 2011). Note, however, that parameterized models cannot account for lateral variations in crustal production, and fully two- or three dimensional models need to be applied in order to resolve young, localized volcanism. Fig. 4 compares the photogeological estimates of Greeley and Schneid (1991) with the results of the numerical models by Grott et al. (2011) and Morschhauser et al. (2011), who as- 
sume melt production in a global melt layer and melt production in localized, hot mantle plumes, respectively. Within uncertainties of the ratio of extrusive to intrusive volcanism, these approaches show satisfactory agreement at intermediate epochs, but the photogeological approach underestimates crustal production rates in the Noachian, whereas the numerical models cannot provide estimates for younger volcanism. Therefore, both approaches complement each other and are necessary for an overall picture of Mars' volcanic history.

As Mars is in the stagnant-lid mode of mantle convection, volatile contents of magmas associated with intra-plate volcanism on Earth have been considered to be comparable to volatile contents on Mars. However, as Mars may have a different volatile content and mantle oxidation state compared to Earth, these values ar at best first-order estimates. However, for lack of better data at the time, terrestrial values fave been assumed in several studies. Today, a better understanding of the differences of the magma volatile content of Earth and Mars exist and a more sophisticated approach will be elaborated at the end of this section. For Mauna Loa and Kilauea on Hawai'i, the concentration of $\mathrm{CO}_{2}$ and $\mathrm{H}_{2} \mathrm{O}$ in the magma is 0.65 wt.\% and 0.30 wt.\%, respectively (Gerlach and Gräber 1985; Greenland $1987 \mathrm{a} ; 1987 \mathrm{~b})$. Subglacial volcanism on Iceland with a $\mathrm{CO}_{2}$ content of $\sim 500 \mathrm{ppm}$ has also been used as an analogue for Mars (O'Neill et al. 2007), although it may differ significantly from the type of volcanism expected in the stagnant-lid regime of mantle convection. Using Hawai'ian volcanism as an analogue for Mars and by neglecting atmospheric escape, Phillips et al. (2001) concluded that 1.5 bar of $\mathrm{CO}_{2}$ have been outgassed during the formation of the Tharsis bulge including intrusions, probably leading to a strong greenhouse effect and climate transition at the end of the Noachian. Since the mid-Noachian, extrusive volcanism may have outgassed an atmosphere of 800 mbar, consisting of 400 mbar $\mathrm{CO}_{2}, 8 \mathrm{~m} \mathrm{EGL} \mathrm{H}_{2} \mathrm{O}$ and 6 other minor species as has been estimated from the photogeological record and Hawai'ian volatile concentrations (Craddock and Greeley 2009).

Being of igneous origin, the volatile content of the Martian meteorites may also serve as a proxy for magma volatile contents. An analysis of melt inclusions and a reconstruction of SNC solidifcation history results in a magma water content of $1.4-1.8$ wt.\% prior to degassing (McSween and Harvey 1993; McSween et al. 2001; Johnson et al. 1991). As an upper bound, the formation of Tharsis could have outgassed $120 \mathrm{~m}$ EGL $\mathrm{H}_{2} \mathrm{O}$ in this way, if a magma volatile content of 2 wt.\% $\mathrm{H}_{2} \mathrm{O}$ is assumed (Phillips et al. 2001). However, water content may have been overestimated and values change to less than $0.3 \mathrm{wt} . \%$ if the high chlorine content in Martian meteorites is taken into account (Filiberto and Treiman 2009). These lower water concentrations are also supported by direct measurements in kaersutitic and biotitic melt inclusions (Watson et al. 1994) and would decrease the amount of water outgassed by Tharsis to $18 \mathrm{~m}$ EGL.

It may be argued that the magma water content of the SNC meteorites, which are believed to be younger than $\sim 1.3 \mathrm{Gyr}$, do not represent the typical magma water content at the time of Tharsis formation $\sim 3$ Gyr ago. As numerical models predict a total mantle water loss of $\sim 50 \%$ due to volcanic outgassing, the uncertainty associated in using SNC magma water contents for Tharsis outgassing is around a factor of two. Compared with other uncertainties, e.g. the volume of Tharsis or the debate on the water magma concentration of the SNCs, this uncertainty is not significant. 


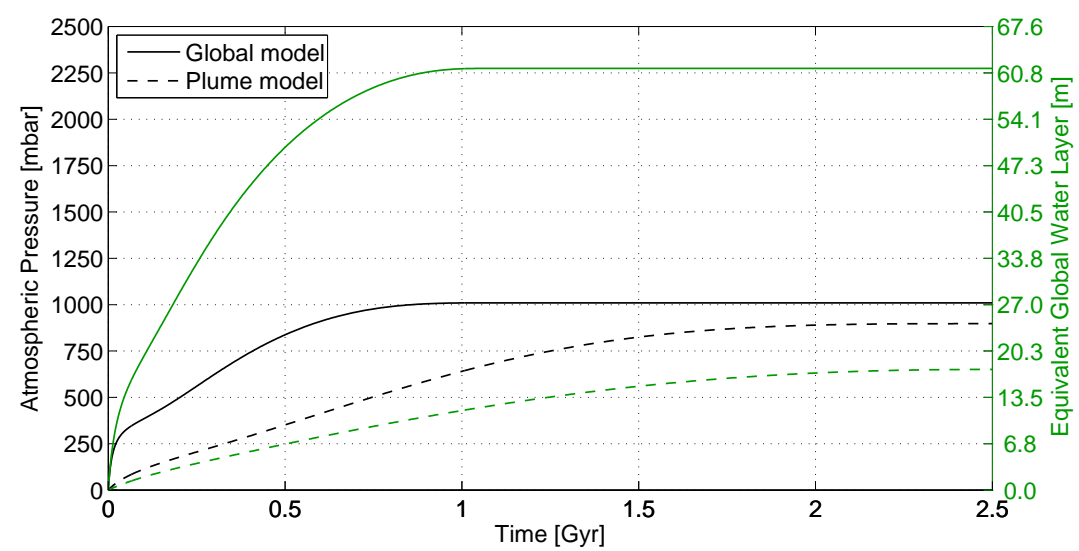

Fig. 5 Modeled cumulative volcanic outgassing of $\mathrm{CO}_{2}$ given as partial surface pressure in mbar (black lines) and of $\mathrm{H}_{2} \mathrm{O}$ given as equivalent global water layer (green lines) as a function of time. Mantle oxygen fugacity was assumed to be one order of magnitude above the ironwustite buffer, resulting in an upper limit on $\mathrm{CO}_{2}$ pressure. Initial mantle water concentration was assumed to be $100 \mathrm{ppm}$. Solid curves correspond to a model considering mantle melting in a global melt channel, whereas dashed curves correspond to a model considering melting in mantle plumes covering only a small fraction of the planetary surface. The outgassing efficiency $\eta$ was set to 0.4 for all models and atmospheric escape is neglected.

In addition, partitioning of water into the melt can be calculated from a melting model. Accumulated fractional melting may be more appropriate for mantle melting on Mars (Fraeman and Treiman 2010; Grott et al. 2012, this issue), but batch melting is also widely applied (Hauck et al. 2002; Morschhauser et al. 2011). However, the difference between the two approaches is comparatively small and results are not significantly affected. Within the frameworks of batch- and fractional melting, the partioning coefficient, melt fraction, and bulk water content determine the concentration in the melt. The partition coefficient of water is most likely close to 0.01 (Katz et al. 2003), and melt fractions obtained from numerical models average around 5-10\% (Hauck et al. 2002; Morschhauser et al. 2011). These values are consistent with melt fractions determined from trace-element analysis of shergottites, which range from $2 \%$ to $10 \%$ (Norman 1999; Borg and Draper 2003) and result in magma water concentrations of 10 to 16 times the bulk water content. The bulk mantle water content of Mars is poorly constrained, and a large range of concentrations have been obtained from different methods: Analysing water content in melt inclusions of Martian meteorites, a bulk mantle water concentration of $1400 \mathrm{ppm}$ was calculated (McSween and Harvey 1993), while numerical accretion models (Lunine et al. 2003) arrive at maxiumum concentrations of $800 \mathrm{ppm}$. In contrast, meteoritic mixing models constrained from element ratios in SNC meteorites predict bulk water concentrations of only $36 \mathrm{ppm}$ (Wänke and Dreibus 1994). It should be noted that, even though the mantle is dehydrating with time and SNCs are believed to be geologically young, the inferred bulk mantle water content of the SNCs is larger than that predicted by the other methods. This may be due to the large uncertainties associated with each of these methods. 
The solubility of $\mathrm{CO}_{2}$ in Martian magma can be calculated by considering the underlying chemistry. For $\mathrm{CO}_{2}$, solubility depends on the form in which graphite is stable in the Martian mantle, which in turn depends on oxygen fugacity (Hirschmann and Withers 1998). The Shergottites, which most likely reflect conditions at the magma source region (Hirschmann and Withers 1998), have oxygen fugacities between the iron-wustite (IW) buffer and one $\log _{10}$ unit above it $(\mathrm{IW}+1)$ (Herd et al. 2002; Shearer et al. 2006). The oldest Martian meteorite, ALH84001, indicates even more reducing conditions around IW-1 (Warren and Kallmeyen 1996). Under these reducing conditions, carbon is stable in the form of graphite (Hirschmann and Withers 1998), and a chemical model for $\mathrm{CO}_{2}$ solubility under graphite saturated conditions (Holloway et al. 1992; Holloway 1998) can be applied (Hirschmann and Withers 1998). Chemical equilibrium constants controlling $\mathrm{CO}_{2}$ solubility have been calibrated using terrestrial basaltic magmas (Holloway et al. 1992) and Martian-basalt analogue material (Stanley et al. 2011), as a function of oxygen fugacity. At relatively oxidizing conditions $(\mathrm{IW}+1)$ and melt fractions typically encountered in the Martian mantle (5-10\%), a maximum of $\sim 1000 \mathrm{ppm} \mathrm{CO}_{2}$ can be dissolved (Grott et al. 2011; Hirschmann and Withers 1998), which is significantly less than the 0.65 wt.\% obtained for Kilauea basalts.

Combining the chemical model for $\mathrm{CO}_{2}$ solubility (Hirschmann and Withers 1998) with parameterized thermal evolution models (Morschhauser et al. 2011), the amount of outgassed $\mathrm{CO}_{2}$ can be calculated self-consistently (Grott et al. 2011). In order to cover the range of expected mantle dynamics in a one-dimensional model, two end member melting models may be considered: Melting in a global melt channel is likely representative for early martian evolution, whereas melting in localized mantle plumes may be more appropriate for the later evolution. Outgassing for both models is shown in Fig. 5. The degree of partial melting encountered in the plume model is generally higher than that for the melt channel model, and volatile concentrations in the melt are therefore lower (cf. Fig. 5). As approximately the same amount of crust is extracted from the mantle in both models, this results in reduced outgassing efficiencies. In both cases, a total of $\sim 1$ bar $\mathrm{CO}_{2}$ can be outgassed if comparatively oxidizing conditions (IW+1) are assumed, and for an initial mantle water concentration of $100 \mathrm{ppm}$ a total of 61 and $18 \mathrm{~m}$ EGL of $\mathrm{H}_{2} \mathrm{O}$ can be outgassed in the global melt channel and plume model, respectively. While the rate of outgassing is lower for the plume model, outgassing in this model persists for $\sim 1$ Gyr after melt generation ceases in the global melt channel model. A parameterization of $\mathrm{CO}_{2}$ and $\mathrm{H}_{2} \mathrm{O}$ outgassing rates as a function of oxygen fugacity, initial mantle water content, and outgassing efficiency may be found in Grott et al. (2011).

From these considerations one can see that, depending on geochemical and geological constraints, early Mars could have accumulated a secondary $\mathrm{CO}_{2}$ atmosphere by volcanic outgassing of $\leq 1$ bar $\sim 4$ Gyr ago. However, large impacts and atmospheric escape processes should have modified the growth of this secondary atmosphere. In the following sections we will investigate possible changes of the secondary atmosphere in relation to losses and sources caused by large impacts, as well as various atmospheric escape processes which are connected to the change in solar activity. 


\section{Atmosphere Erosion and Delivery by Large Impacts}

Atmospheric erosion and delivery by impactors can be studied with the help of hydrocode simulations which essentially simulate the flow field and dynamic response of materials by taking into account material strength and rheology (Pierazzo and Collins 2003; Shuvalov and Artemieva 2001; Svetsov 2007, Melosh and Vickery 1989). Previous atmospheric erosion studies by hydrocodes have not always provided similar results, mainly due to differences in the physical models such as the choice of an appropriate equation of state, or a proper model of vapor cloud dynamics (Pham et al. 2009). In addition, these simulations require very large computer resources and can not be used directly to simulate long term atmospheric evolution. Therefore, the influence of the major parameters on atmospheric erosion and delivery has been parameterized. Models using the parameterization of the major mechanisms affecting the atmospheric erosion and delivery by the impacts can be instead used to study the evolution of the atmosphere. Many studies applied the so-called "tangent plane model" which has been developed by Melosh and Vickery (1989).

The tangent plane model of Melosh and Vickery (1989) is based on their hydrocode simulation results. Their model has been modified to take into account other simulations as well as additional parameters, and has been used to obtain a global view of the atmospheric mass evolution (Zahnle et al. 1992; Zahnle 1993; Manning et al. 2006; 2009; Pham et al. 2009). The advantage of using analytical models is that they can represent basic aspects of impact erosion and delivery, while reducing computation time since they only use a reduced number of parameters, scaled with numerical hydrocode simulation results.

The principle of the tangent plane model is that, when an impactor above a critical mass, $m_{\text {crit }}$, strikes the planet, the total mass above the plane tangent to the surface at the impact point, $m_{\mathrm{tan}}$, escapes.

The critical mass is the minimal impactor mass that can eject $m_{\mathrm{tan}}$, and it is proportional to $m_{\text {tan }}$ through a factor $n$ which represents the impact efficiency. The atmospheric mass above the plane tangent of the impact surface is approximated by $m_{\tan }=m_{\mathrm{atm}} H / 2 R_{\mathrm{pl}}$, assuming an isothermal atmosphere in hydrostatic equilibrium, where $m_{\mathrm{atm}}$ is the total atmospheric mass, $H$ the atmospheric scale height, and $R_{\mathrm{pl}}$ the radius of the planet. Note that the tangent plane model is only an approximation of erosion and delivery processes and that small impactors can still remove atmosphere (Zahnle, 1993). While the model doesn't reproduce the physics of impact erosion or delivery, it can, with a suitable parameterization of the critical mass, give a global view of the atmospheric mass evolution upon impacts with a minimum set of variables (related to the critical mass value) and a much smaller computation time. The total mass evolution $M_{\mathrm{atm}}$ is controlled by the difference between the rates of change of atmospheric erosion, $M_{\mathrm{esc}}$ and the delivered volatile mass, $M_{\text {del }}$

$$
\frac{d M_{\mathrm{atm}}}{d t}=\frac{d M_{\mathrm{del}}}{d t}-\frac{d M_{\mathrm{esc}}}{d t}
$$

with

$$
\frac{d M_{\mathrm{esc}}}{d t}=\frac{\partial N_{\mathrm{cum}}\left[>m_{\mathrm{crit}}(t), t\right]}{\partial t} 4 \pi R^{2} m_{\mathrm{tan}}(t) f_{\mathrm{vel}} f_{\mathrm{obl}}
$$




\begin{tabular}{c|c|c|c}
\hline Factor & Asteroids & \multicolumn{2}{|c}{ Comets } \\
& & SP comets & LP comets \\
\hline$f_{\text {vel }}$ & 0.08 & 0.83 & 0.99 \\
$f_{\text {obl }}$ & 7.54 & \multicolumn{2}{|c}{2.16} \\
$y_{\text {imp }}$ & 0.01 & \multicolumn{2}{|c}{0.3} \\
$f_{\text {vap }}$ & 0.34 & \multicolumn{2}{|c}{1} \\
$g_{\text {vap }}$ & 0.21 & \multicolumn{2}{|c}{1} \\
\hline
\end{tabular}

Table 1 Values of the factors given in Eqs. (6) and (7).

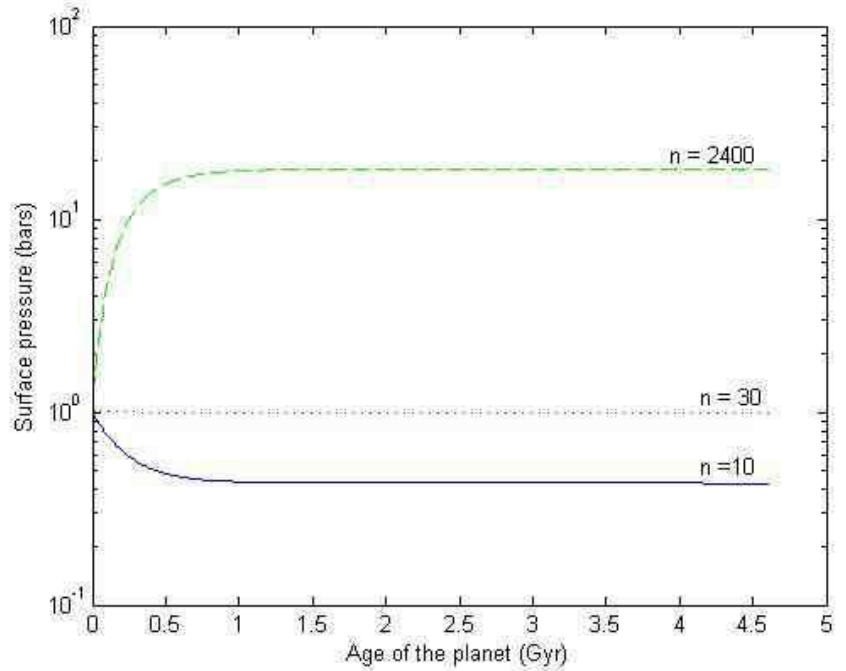

Fig. 6 Maximum diameter of the impactors hitting Mars as a function of time, for an assumed initial atmospheric surface pressure of $300 \mathrm{mbar}$. The horizontal lines show the critical diameter $d_{\text {crit }}$ for different values of $n=m_{\text {crit }} / m_{\text {tan }}$ (details in the main text). Only impactors with diameter larger than $d_{\text {crit }}$ can erode the atmosphere. The lower limit of $n=10$ represents an extreme case corresponding to the upper limit of atmospheric erosion.

and

$$
\begin{gathered}
\frac{d M_{\mathrm{del}}}{d t}=\frac{\partial N_{\mathrm{cum}}\left[>m_{\mathrm{crit}}(t), t\right]}{\partial t} 4 \pi R^{2} \frac{b}{1-b} m_{\text {crit }}(t) y_{\text {imp }} f_{\mathrm{vap}}+ \\
\frac{\partial N_{\mathrm{cum}}\left[>m_{\mathrm{crit}}(t), t\right]}{\partial t} 4 \pi R^{2} m_{\text {crit }}(t) y_{\text {imp }}\left(1-f_{\mathrm{vel}} f_{\mathrm{obl}} g_{\mathrm{vap}}\right) .
\end{gathered}
$$

where $N_{\text {cum }}$ is the cumulative number of impacts with mass larger than $m_{\text {crit }}$ at a time $t, \partial N_{\text {cum }}\left(>m_{\text {crit }}(t), t\right) / \partial t$ represents the flux of these impactors and $b$ characterizes the mass distribution of the impactor flux, $b<1$. We also assumed an exponentially decaying impact flux (e.g., Neukum and Wise 1976; Ivanov 2001; Neukum et al. 2001).

In the above equations the original "tangent plane model" is modified by the additional terms $f_{\text {vel }}$ and $f_{\text {obl }}$ in Eq. 5 and $y_{\text {imp }} f_{\text {vap }}, g_{\text {vap }}, f_{\text {vel }}$ and $f_{\text {obl }}$ in Eq. 6] which were not considered in previous studies (Pham et al. 2009; 2011). The 
fraction of impactors which are fast enough to erode the planet $\left(f_{\mathrm{vel}}\right)$ as well as the enhancement factor of the erosion due to impact obliquity $\left(f_{\mathrm{obl}}\right)$ are taken account. The volatile content $\left(y_{\mathrm{imp}}\right)$ is different for asteroids and comets. We assume a volatile content of $y_{\mathrm{imp}}=0.03$ for comets, and $y_{\mathrm{imp}}=0.01 \mathrm{wt}$. for asteroids. The ratio of the vaporized mass to the impactor mass averaged over impact velocities is considered through the parameters $f_{\text {vap }}$ or $g_{\text {vap }}$ depending on whether the impactor mass is below or above the critical mass, respectively. In the delivery equation, the first term on the right side represents the delivered mass rate for $m_{\text {imp }}<$ $m_{\text {crit }}$, and the second term is the delivered mass rate for the fraction of impactors with $m_{\text {imp }}>m_{\text {crit }}$ that was not removed by impacts. The relative amount of comets and asteroids in the total impact flux on Mars is assumed to be $6 \%$ and 94\%, respectively, as suggested by Olsson-Steel (1987). In addition comets are differentiated between short-period (SP) comets $(\sim 4 \%)$ and long-period comets (LP) from the Oort cloud $(\sim 2 \%)$ when the impact velocity is taken into account in the simulations. The values corresponding to the factors given in Eq. 5 and Eq. 6] are shown in Table 2.

The efficiency of atmospheric erosion and delivery is determined by the factor $n$ which is given by $n=m_{\text {crit }} / m_{\text {tan }}$. The exact value of $n$ in the tangent plane model is uncertain. The value suggested initially by Melosh and Vickery (1989), $n=1$, has been revised by more recent studies to $n=10$ (Vickery 1990; Manning et al. 2006). On the other hand, the more recent hydrocode simulations performed by Shuvalov and Artemieva (2001), Ivanov et al. (2002), and Svetsov (2007) yield results compatible with much larger values of the critical mass, on the order of $50<n<2000$. Note that $n$ is a function of atmospheric pressure (Hamano and Abe 2006; Svetsov 2007). Pham et al. (2009) considered the tangent plane model with different impact erosion efficiencies, using constant as well as pressure-dependent values of $n$.

The total atmospheric mass evolution calculated by using Eqs. 5 and 6 is plotted in Fig. 5 for three values of $n$. Depending on the value of $n$ impacts constitute either a factor of erosion or a source of volatiles on Mars. The most recent hydrocode simulations tend to favor larger values, $n>30$, for which impacts are a source of volatile (Svetsov, 2007). Although impacts can remove atmosphere for smaller values of $n$ the process is not very efficient, since even for the most favorable values of $n=10$, a 1 bar protoatmosphere can not be eroded to present values over the age of the Solar System. In Sect. 6 we will consider the most favorable parameters for atmospheric loss considering a large impact flux during the late heavy bombardment with lower limit of $n$ to yield an upper limit of atmospheric erosion. Although the impact erosion may not have been a very relevant loss process the question remains if the impact flux for accumulation of a secondary atmosphere was higher than the expected EUV-powered thermal escape flux which will be discussed in Sect. 5 .

\section{The EUV-Powered Blow-Off of the Protoatmosphere and the Change of a Secondary $\mathrm{CO}_{2}$ Atmosphere by Escape Processes}

From observations of young solar-type G stars it is known that despite a weaker total luminosity, stars with a young age are a much stronger source of X-rays and EUV electromagnetic radiation (e.g., Newkirk 1980; Skumanich and Eddy 1981; 
Zahnle and Walker 1982; Güdel et al. 1997). Since the 90ies the evolution of UV fluxes of a sample of solar analogue stars, so-called proxies of the Sun have been studied in detail by spectral measurements from the IUE satellite (Dorren and Guinan 1994). This research was extended by Güdel et al. (1997), Ribas et al. (2005) and recently by Claire et al. 2012 to X-rays and EUV. The wavelength range $\lambda \leq 1000 \AA$ is relevant for ionization, dissociation and thermospheric heating (e.g., Hunten $1972 ; 1987 ; 1993)$.

Because X-rays dominate at young stellar ages, Owen and Jackson (2012) studied the contribution of harder X-ray's to the heating of hydrogen-rich upper atmospheres. If we compare the X-ray luminosities of solar proxies with younger age (e.g. Ribas et al. 2005; Claire et al. 2012) at Mars' orbit, with the values necessary for having a dominating X-ray driven atmospheric escape (Owen and Jackson 2012, their Fig. 11), one finds that this process is only relevant for hydrogen-rich "Hot Jupiter"-type exoplanets but can be neglected on early Mars which orbits further away from the Sun. Therefore, for early Mars, EUV radiation should be the main heating process in the thermosphere.

Güdel et al. (1997), Ribas et al. (2005) and Claire et al. 2012 analyzed multiwavelength EUV observations by the ASCA, ROSAT, EUVE, FUSE and IUE satellites of solar proxies with ages $<4.6 \mathrm{Gyr}$ and found that the EUV flux is saturated during the first $100 \mathrm{Myr}$ at a value 100 times that of the present Sun. As shown in Fig. 7 this early active period of the young Sun decreases according to an EUV enhancement factor power law after the first $100 \mathrm{Myr}$ (Ribas et al. 2005)

$$
S_{\mathrm{EUV}}=\left(\frac{t_{\mathrm{Gyr}}}{t_{0}}\right)^{-1.23},
$$

where $t_{0}$ is the age of the present Sun and $t_{\mathrm{Gyr}}$ the younger or older age of the Sun in time in units of Gyr.

Due to the lack of accurate astrophysical observations from solar proxies with different ages, previous pioneering studies on EUV-driven hydrodynamic escape of primitive atmospheres were based only on rough EUV enhancement scaling factors which were assumed to be similar or up to only $\sim 5-25$ times higher than the present value (e.g., Watson et al. 1981; Kasting and Pollack 1983; Chassefière 1996). Furthermore, most of the previous studies are based on terrestrial planet formation models where the accretion for Mars occurred quite late at $~ 100 \mathrm{Myr}$ (Wetherill 1986).

Because Mars can be considered as a planetary embryo that did not collide or merge with other embryos it developed most likely within $\sim 2-4$ Myr after the birth of the Solar System (Dauphas and Pourmand 2011; Brasser 2012). This age agrees with average planetary nebula evaporation time scales of $\sim 3 \mathrm{Myr}$. Because nebulae life times are <10 Myr (e.g., Lunine et al. 2011), the nebula-based hydrogen-rich martian protoatmosphere was most likely exposed during several tens of Myr or up to 150 Myr to an EUV flux which was 50-100 times higher compared to today's Sun (Ribas et al. 2005; Claire et al. 2012). Because of the high EUV flux of the young Sun $\mathrm{H}_{2}, \mathrm{H}_{2} \mathrm{O}$ and most $\mathrm{CO}_{2}$ molecules in the thermosphere are dissociated and $\mathrm{H}$ atoms should dominate the upper atmosphere until they escaped to space.

Depending on the composition of the upper atmosphere and the planet's mean density, when the solar EUV flux in the wavelength range $\lambda \approx 2-120$ nm overcomes a critical value, the outward flow of the bulk thermosphere cools due to adiabatic expansion (Tian et al. 2005; 2008). According to studies of Watson et al. (1981), 


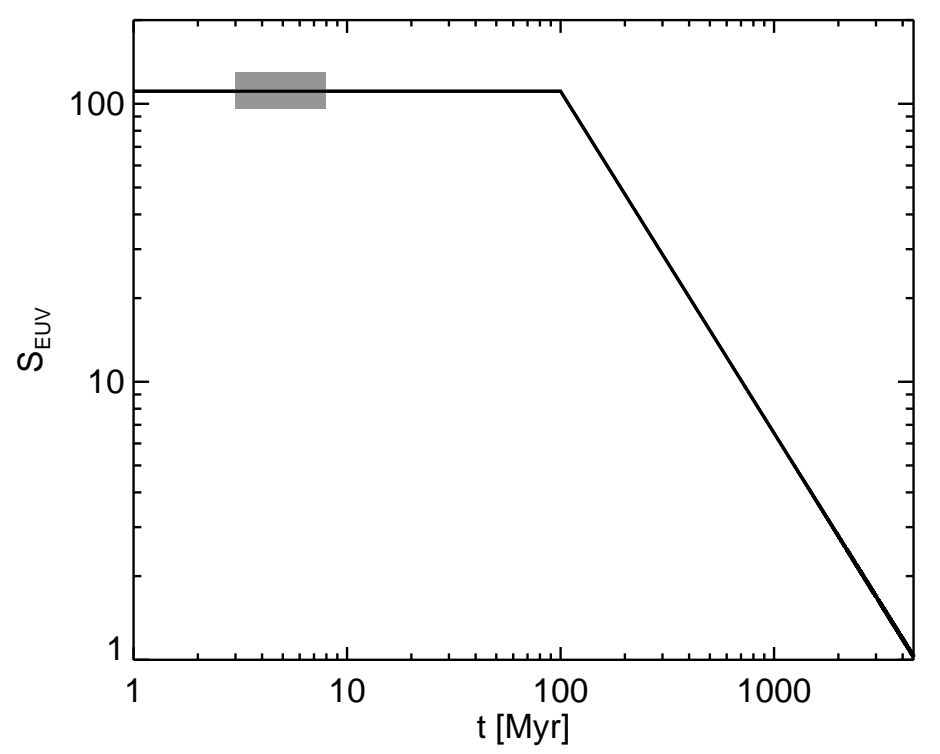

Fig. 7 Solar EUV flux enhancement factor $S_{\mathrm{EUV}}$ as obtained from observations of solar proxies. The average nebula evaporation time is $\sim 3$ Myr. Since that time period planetary embryos and protoplanets are exposed to the saturated EUV flux value which is $\sim 100$ times larger compared to the present solar value for about 90 Myr. The shaded area marks the expected time when Mars ended its accretion.

Kasting and Pollack (1983) and Tian et al. (2005), if hydrogen populates the upper atmosphere of a terrestrial planet, its exobase level can expand several planetary radii if the EUV flux is only a few times higher compared to that of today's Sun. A hydrogen-rich upper atmosphere of a martian-type body which is exposed to a EUV flux which is 5-100 times higher compared to the present solar value is, therefore, certainly in the blow-off regime. Under such conditions the exosphere evaporates as long as enough hydrogen is present.

By applying a blow-off formula which is derived from the energy-limited equation (e.g., Hunten 1987; 1993) the atmospheric mass loss $d M_{\mathrm{esc}} / d t$ can be written as

$$
\frac{d M_{\mathrm{esc}}}{d t}=\frac{3 \eta S_{\mathrm{EUV}} F_{\mathrm{EUV}}}{4 G \rho_{\mathrm{pl}}}
$$

where the heating efficiency $\eta$ is the ratio of the net heating rate to the rate of solar EUV energy absorption of 15-40\% (Chassefiere 1996; Lammer et al. 2009; Koskinen et al. 2012), the gravitational constant $G$, the mean planet density $\rho_{\mathrm{pl}}$ and the present time EUV flux $F_{\mathrm{EUV}}$ in Mars' orbit. Using this relation one can estimate the atmospheric escape as long as hydrodynamic blow-off conditions occur which means that the thermal energy of the gas kinetic motion overcomes the gravitational energy.

Fig. 8a shows the upper limit of a EUV-driven hydrogen-dominated protoatmosphere, which may have been captured from the nebula, during $\sim 1$ Gyr after the planet's origin. The loss is estimated from Eq. (8) with a heating efficiency $\eta$ of $40 \%$ (Koskinen et al. 2012). One can see that Mars could have lost an equivalent 
hydrogen content as available in $\sim 14$ Earth oceans $\left(\mathrm{EO}_{\mathrm{H}}\right)$. Due to the low gravity of Mars and EUV fluxes on the order of $\geq 50$ times that of the present Sun, the blow-off condition was $100 \%$ fulfilled for light hydrogen atoms and most likely also for heavier atomic species such as $\mathrm{O}$, or $\mathrm{C}$ if they populated the upper atmosphere (e.g., Tian et al. 2009).

After the dissociation of the $\mathrm{H}_{2} \mathrm{O}$ molecules and a fraction of $\mathrm{CO}_{2}$ one can expect that $\mathrm{O}$ atoms are the major form of escaping oxygen. The escape flux of the heavier atoms $F_{\text {heavy }}$ which can be dragged by the dynamically outward flowing hydrogen atoms with flux $F_{\mathrm{H}}$ can be written as (e.g., Hunten et al. 1987; Chassefière 1996)

$$
F_{\text {heavy }}=\frac{X_{\text {heavy }}}{X_{\mathrm{H}}} F_{\mathrm{H}}\left(\frac{m_{\mathrm{c}}-m_{\text {heavy }}}{m_{\mathrm{c}}-m_{\mathrm{H}}}\right) \text {, }
$$

where $X_{\mathrm{H}}$ and $X_{\text {heavy }}$ are the mole mixing ratios. $m_{\mathrm{H}}$ and $m_{\text {heavy }}$ are the masses of the hydrogen atom and the heavy species. $m_{\mathrm{c}}$ is the so called cross over mass

$$
m_{\mathrm{c}}=m_{\mathrm{H}}+\left(k T F_{\mathrm{H}}\right)\left(b g X_{\mathrm{H}}\right),
$$

which depends on $F_{\mathrm{H}}$, a molecular diffusion parameter $b$ (Zahnle and Kasting 1986; Chassefiére, 1996), the gravity acceleration $g$, Boltzmann constant $k$ and an average upper atmosphere temperature $T$, which can be assumed under such conditions for hydrogen to be on the order of $\sim 500 \mathrm{~K}$ (Zahnle and Kasting 1986; Chassefiére 1996). By applying Eqs. 1 and 2 and assuming that Mars finished its accretion
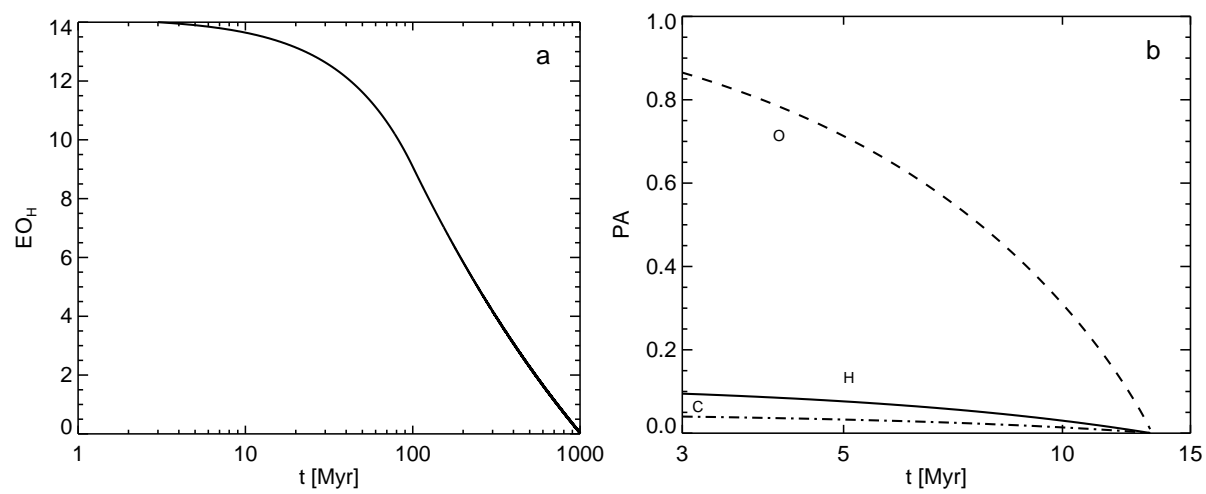

Fig. 8 a: Upper escape value of atomic hydrogen in units of Earth ocean equivalent amounts $\left(\mathrm{EO}_{\mathrm{H}}\right)$ of atomic hydrogen from a martian protoatmosphere between 3 Myr to 1 Gyr after the origin of the Solar System. b: Calculated normalized loss of an outgassed 70 bar water vapor and 12 bar $\mathrm{CO}_{2}$ steam atmosphere as a function of time. $\mathrm{PA}=1$ corresponds to the total pressure of 82 bar, while the solid line corresponds to loss of the hydrogen content. The dashed and dash-dotted lines corresponds to dragged oxygen and carbon atoms which originate from dissociation of $\mathrm{H}_{2} \mathrm{O}$ and $\mathrm{CO}_{2}$.

within the EUV-saturated epoch of the young Sun, Fig. 8b shows the atmosphere loss estimation of an outgassed water vapour dominated by a 70 bar $\mathrm{H}_{2} \mathrm{O}$ and 12 bar $\mathrm{CO}_{2}$ steam atmosphere. The loss is normalized to the total outgassed surface pressure of 82 bar, where $\mathrm{PA}=1$ which corresponds to the total pressure of 82 bar. One can see that under such conditions early Mars could easily lose 
its initial atmosphere in $\sim 10$ Myr. Thus, because Mars accreted early (Dauphas and Pourmand 2011; Brasser 2012), even if the planet would have obtained its volatile inventory later, the high EUV flux of the young Sun would have blown the atmosphere away. One can also see that under these extreme conditions the outgassed $\mathrm{CO}_{2}$ would be lost, either in dissociated form as $\mathrm{C}$ and $\mathrm{O}$ as shown in Fig. 8b. Therefore, a dense $\mathrm{CO}_{2}$ atmosphere could have been lost very early and could not have accumulated during the early Noachian. On the other hand, if early Mars was surrounded by a nebula-based hydrogen envelope, the outgassed heavier volatiles may have been protected against atmospheric escape until the captured hydrogen was lost and did not dominate the upper atmosphere anymore.

For these time scales the outgassed $\mathrm{H}_{2} \mathrm{O} / \mathrm{CO}_{2}$ atmosphere remained most likely in steam form because the time scale where the surface temperatures may reach the point that $\mathrm{H}_{2} \mathrm{O}$ can condense is comparable (Elkins-Tanton 2008). Furthermore, these time scales also agree with studies by several researchers who investigated the early stages of accretion and impacts and expect that due to thermal blanketing hot temperatures could keep the volatiles in vapor phase for several tens of Myr or even up to 100 Myr (e.g., Hayashi et al. 1979; Mizuno et al. 1980; Matsui and Abe, 1986; Zahnle et al. 1988; Abe 1997; Albarède and Blichert-Toft 2007). One can also see from Fig. 5 and Fig. 6 and the discussions in Sect. 4 that during this early evolutionary period most of the impacts occurred. Although the loss effect of these impacts may have been not so efficient when Mars had a dense outgassed $\mathrm{H}_{2} \mathrm{O} / \mathrm{CO}_{2}$ steam atmosphere. As mentioned in Sect. 4 impacts have contributed to a permanent heating of the atmosphere. On the other hand volatiles which were brought in to the atmosphere by large impacts should also have been lost due to the strong hydrogen escape.

These results are in agreement with the non-detection of carbonates by the OMEGA instrument on board of ESA's Mars Express spacecraft (Bibring et al. 2005). Mars Express mapped a variety of units based on areas exhibiting hydrated minerals, layered deposits, fluvial floors, and ejecta of deep craters within Vastitas Borealis with a surface resolution in the $\sim 1-3-\mathrm{km}$ range. Besides $\mathrm{CO}_{2}$-ice in the perennial southern polar cap, no carbonates were reported. Bibring et al. (2005) concluded that the non-detection of carbonates would indicate that no major surface sink of $\mathrm{CO}_{2}$ is present and the initial $\mathrm{CO}_{2}$, if it represented a much higher content, would then have been lost from Mars early rather than stored in surface reservoirs after having been dissolved in long-standing bodies of water.

However, so far it is not clear when the outgassing flux from Mars interior exceeded the expected escape flux so that a secondary $\mathrm{CO}_{2}$ atmosphere could grow during the later Noachian. It should be noted that the accumulation of both the secondary outgassed atmosphere and volatiles which were possibly delivered by later impacts is highly dependent on atmospheric escape after the strong early hydrodynamic loss during the EUV-saturation phase of the young Sun.

Tian et al. (2009) applied a 1D multi-component hydrodynamic thermosphereionosphere model and a coupled electron transport-energy deposition model to Mars and found that for EUV fluxes $>10$ times that of today's Sun, $\mathrm{CO}_{2}$ molecules dissociate efficiently resulting in less IR-cooling of $\mathrm{CO}_{2}$ molecules in the thermosphere so that a $\mathrm{CO}_{2}$ atmosphere was most likely also not stable on early Mars after the EUV-saturation phase ended. According to this study, the flux of the produced $\mathrm{C}$ and $\mathrm{O}$ atoms is $>10^{11} \mathrm{~cm} \mathrm{~s}^{-1}$ before $\sim 4$ Gyr ago and was of the same order as the fluxes from volcanic outgassing (Tian et al. 2009). 
Although this result seems logical, because the results of Tian et al. (2009) are model dependent and contain various uncertainties we estimate the possible growth of such a secondary $\mathrm{CO}_{2}$ atmosphere from the outgassing rates shown in Fig. 5 or Grott et al. (2011), by considering that the outgassed $\mathrm{CO}_{2}$ flux exceeded the thermal escape since 4.3, 4.2, 4.1 or 4 Gyr ago. Fig. 9 shows the possible sce-
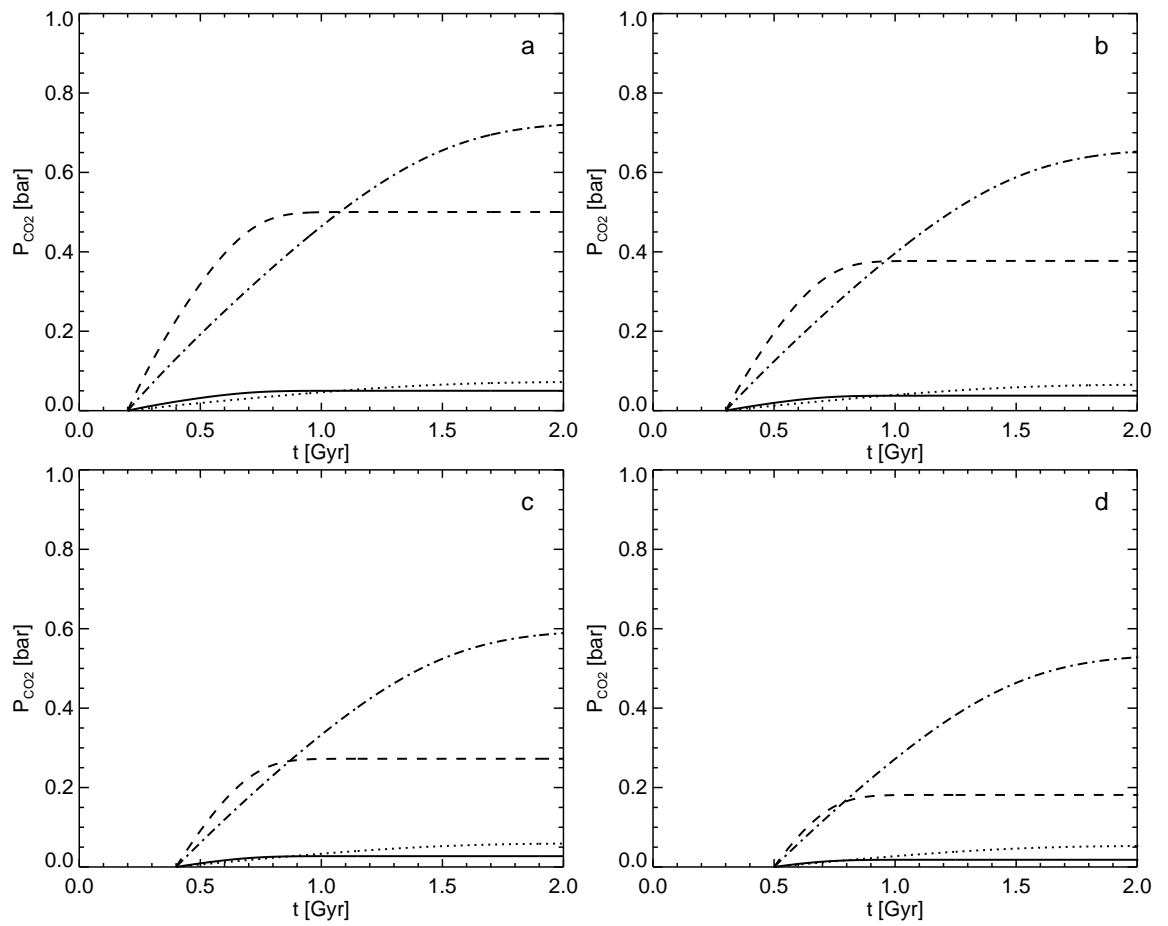

Fig. $9 \mathrm{CO}_{2}$ partial surface pressure as a function of time with the same assumptions as in Fig. 4 but for various onset times for the build up of a secondary $\mathrm{CO}_{2}$ atmosphere after total loss of the earlier outgassed $\mathrm{CO}_{2}$ content. Dashed lines: IW=1, Surface fraction of the melt channel $f_{\mathrm{p}}=1$; dashed-dotted lines: IW $=1, f_{\mathrm{p}}=0.01$; solid lines: IW $=0, f_{\mathrm{p}}=1$; dotted lines $\mathrm{IW}=0$ and $f_{\mathrm{p}}=0.01$. The onset for atmospheric growth of a secondary $\mathrm{CO}_{2}$ atmosphere is assumed in a: 4.3 Gyr (a), 4.2 Gyr (b), 4.1 Gyr (c), and 4 Gyr (d) ago.

narios for a build up of a secondary $\mathrm{CO}_{2}$ atmosphere and Fig. 10 shows secondary outgassed $\mathrm{H}_{2} \mathrm{O}$ amounts in units of bar. Table 3 summarizes the accumulated outgassed $\mathrm{CO}_{2}$ amount in units of bar after the outgassed flux becomes more efficient compared to the escape flux for the same oxygen fugacity the related iron-wustite buffer (IW) and $f_{\mathrm{p}}$ scenarios shown in Fig. 9. If we consider that the escape flux of $\mathrm{CO}_{2}$ was less than that from the interior $4.3 \mathrm{Gyr}$ ago (Fig. 9a) a secondary $\mathrm{CO}_{2}$ atmosphere of $\sim 0.7$ bar could build up $\sim 4$ Gyr ago, which is about 100 times denser than the present atmosphere if one assumes a global melt channel. By considering mantle plumes only, the outgassing would be finished about $4 \mathrm{Gyr}$ ago and a $\mathrm{CO}_{2}$ atmosphere of $\sim 0.5$ bar could have been built up. If the escape flux could balance the volcanic outgassing for longer times, depending if one assumes a global melt channel or mantle plumes, only $\mathrm{CO}_{2}$ atmospheres with lower upper densities of 

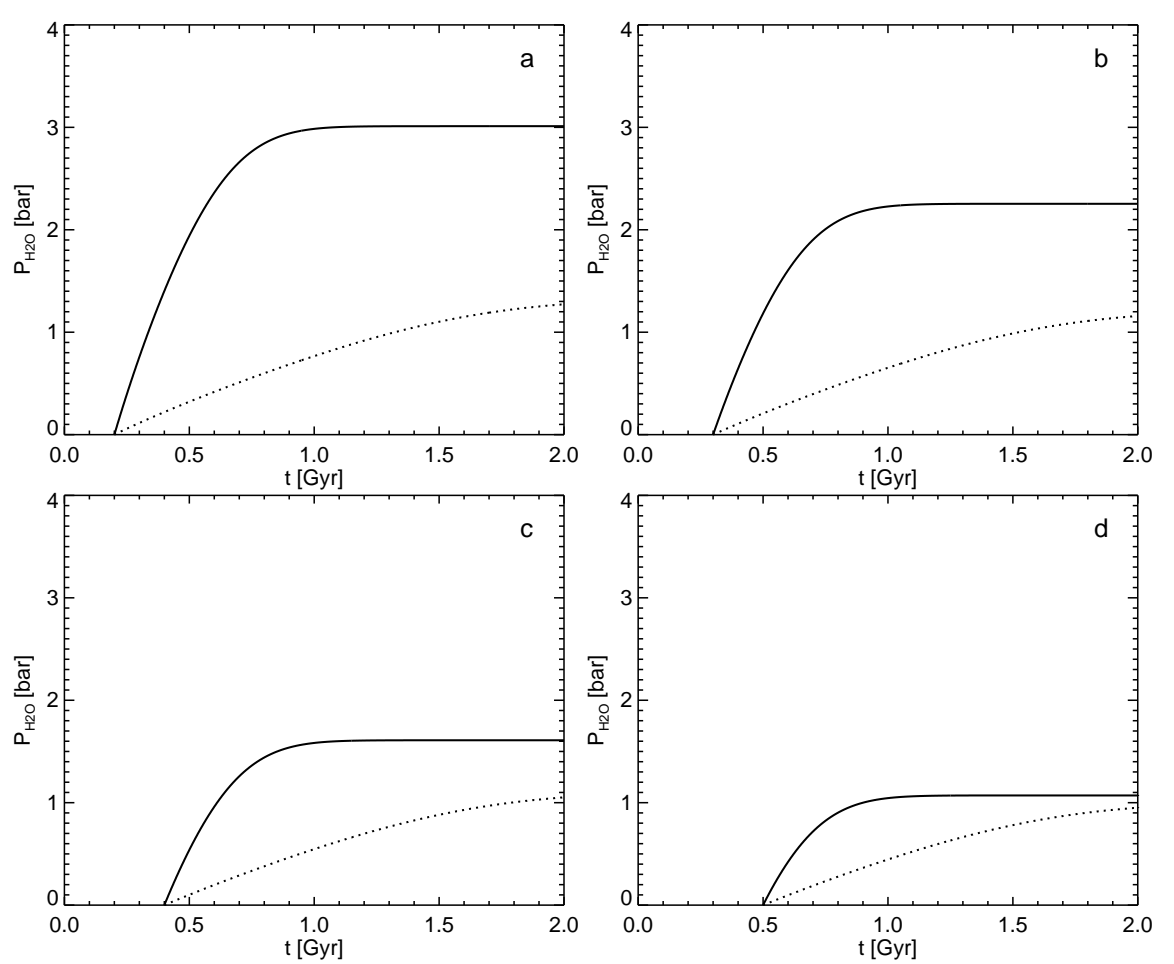

Fig. 10 Outgassed $\mathrm{H}_{2} \mathrm{O}$ in units of bar as a function of time with the same assumptions as in Fig. 4 but for various onset times where the outgassing flux exceeded the escape flux after the total loss of the earlier outgassed water content. Solid lines: Surface fraction of the melt channel $f_{\mathrm{p}}=1$; dotted lines: $f_{\mathrm{p}}=0.01$. The bulk concentration of water in the mantle is assumed to be $100 \mathrm{ppm}$ and the outgassing efficiency is assumed to be 0.4 .

Table 2 Secondary outgassed $\mathrm{CO}_{2}$ partial surface pressure in units of bar as function of ironwustite (IW) buffer and surface fraction of the melt channel $\left(f_{\mathrm{p}}\right)$ (see Sect. 3 and Grott et al. 2011) and time $t$ after Mars' origin in Myr when the outgassing flux is assumed larger than the escape flux.

\begin{tabular}{l|cccc}
\hline IW and $f_{\mathrm{p}}$ scenarios & $t=200 \mathrm{Myr}$ & $t=300 \mathrm{Myr}$ & $t=400 \mathrm{Myr}$ & $t=500 \mathrm{Myr}$ \\
\hline IW $=1 ; f_{\mathrm{p}}=0.01$ & $\sim 0.7 \mathrm{bar}$ & $\sim 0.65 \mathrm{bar}$ & $\sim 0.6 \mathrm{bar}$ & $\sim 0.55 \mathrm{bar}$ \\
IW $=1 ; f_{\mathrm{p}}=1$ & $\sim 0.5 \mathrm{bar}$ & $\sim 0.37 \mathrm{bar}$ & $\sim 0.25 \mathrm{bar}$ & $\sim 0.18 \mathrm{bar}$ \\
IW $=0 ; f_{\mathrm{p}}=0.01$ & $\sim 0.07 \mathrm{bar}$ & $\sim 0.12 \mathrm{bar}$ & $\sim 0.11 \mathrm{bar}$ & $\sim 0.1 \mathrm{bar}$ \\
$\mathrm{IW}=0 ; f_{\mathrm{p}}=1$ & $\sim 0.05 \mathrm{bar}$ & $\sim 0.085 \mathrm{bar}$ & $\sim 0.05 \mathrm{bar}$ & $\sim 0.03 \mathrm{bar}$ \\
\hline
\end{tabular}

$\sim 0.2-0.4$ bar could build up $\sim 2.5-4$ Gyr ago. For cases with low IW-buffers, the secondary $\mathrm{CO}_{2}$ atmosphere would only have a surface density between $\sim 50-100$ mbar.

Low $\mathrm{CO}_{2}$ surface pressure values would also agree with a study by Zahnle et al. (2008), which is based on a photochemical $\mathrm{CO}_{2}$ stability problem discussed by McElroy (1972), that a martian $\mathrm{CO}_{2}$ atmosphere much denser than several 100 
Table 3 Secondary outgassed $\mathrm{H}_{2} \mathrm{O}$ partial pressure in units of bar with similar conditions and assumptions as shown in Fig. 10.

\begin{tabular}{l|cccc}
\hline$f_{\mathrm{p}}$ scenarios & $t=200 \mathrm{Myr}$ & $t=300 \mathrm{Myr}$ & $t=400 \mathrm{Myr}$ & $t=500 \mathrm{Myr}$ \\
\hline$f_{\mathrm{p}}=1$ & $\sim 3 \mathrm{bar}$ & $\sim 2.25 \mathrm{bar}$ & $\sim 1.6 \mathrm{bar}$ & $\sim 1.1 \mathrm{bar}$ \\
$f_{\mathrm{p}}=0.01$ & $\sim 1.3 \mathrm{bar}$ & $\sim 1.2 \mathrm{bar}$ & $\sim 1.1 \mathrm{bar}$ & $\sim 1 \mathrm{bar}$ \\
\hline
\end{tabular}

mbar may be not be stable for a long time because the $\mathrm{CO}_{2}$ will be photochemically converted into $\mathrm{CO}$ over timescales of $\sim 0.1-1$ Gyr.

Chevrier et al. (2007) investigated the geochemical conditions which prevailed on the martian surface during the Noachian period by applying calculations of aqueous equilibria of phyllosilicates. These authors found that $\mathrm{Fe}^{3+}$-rich phyllosilicates most likely precipitated under weakly acidic alkaline $\mathrm{pH}$, which was a different environment compared to the following period which was dominated by strong acid weathering that led to the observed martian sulphate deposits. Chevrier et al. (2007) applied thermodynamic calculations which indicate that the oxidation state of the martian surface should have been also high during early periods, which supports our results of an early efficient escape of hydrogen.

However, equilibrium with carbonates implies that the precipitation of phyllosilicates occurs at low $\mathrm{CO}_{2}$ partial pressure. Thus, from these considerations one would expect that the lower surface $\mathrm{CO}_{2}$ pressure shown in Figs. 8 and Table 3 may have represented the martian atmosphere $\sim 4$ Gyr ago. If geochemical processes prevented the efficient formation of carbonates then a dense $\mathrm{CO}_{2}$ atmosphere could not have been responsible for a long-term greenhouse effect which is necessary to enable liquid water to remain stable at the surface in the post-Noachian period. In such a case other greenhouse gases such as $\mathrm{CH}_{4}, \mathrm{SO}_{2}, \mathrm{H}_{2} \mathrm{~S}$, etc. (Kasting 1997; Johnson et al. 2008) would be needed to solve the greenhouse-liquid water problem during the late Noachian.

Depending on the surface fraction of the melt channel $f_{\mathrm{p}}$ and the onset time of accumulation from Fig. 10 and Table 4 one can see that the outgassed amount of $\mathrm{H}_{2} \mathrm{O}$ by volcanos would correspond to values $\sim 1-3$ bar, that is a $\approx 20-60 \mathrm{~m}$ EGL. One should also note that additionally to the secondary outgassed atmosphere significant amounts of water and carbon may have been brought later by comets. According to Morbidelli et al. (2000), Lunine et al. (2003) the equivalent of $\sim 0.1$ terrestrial ocean of $\mathrm{H}_{2} \mathrm{O}$, that is a $\sim 300 \mathrm{~m}$ deep EGL of water, could have been provided to Earth by comets during the few 100 Myr following main accretion. The net budget of cometary impacts could hypothetically have also resulted in a net accretion of several bars or even tens of bars of $\mathrm{H}_{2} \mathrm{O}$ (and several 100 mbar of $\mathrm{CO}_{2}$ ) coming from infalling comets until the late Noachian or during the LHB as discussed in Sect. 6. A fraction of this impact delivered $\mathrm{H}_{2} \mathrm{O}$, if all was not lost due to the high thermal escape rate (e.g. Tian et al. 2009) could be stored in the crust (Lasue et al. 2012; Niles 2012). Although it is not clear at the present how much $\mathrm{CO}_{2}$ was in the martian atmosphere $\sim 4$ Gyr ago, the secondary outgassed and accumulated atmosphere was most likely denser compared to the 7 mbar of today. 


\section{Environmental Effects of the Late Heavy Bombardment}

Although the previous sections have shown that due to the high EUV flux of the young Sun, atmospheric escape models do not favor a dense $\mathrm{CO}_{2}$ atmosphere during the first Gyr, we now investigate possible effects of the late heavy bombardment (LHB) period. The ratios between critical mass vs. tangent mass $n$ as discussed in Sect. 4 determines whether impacts cause atmospheric erosion or if they are rather a source of volatile. For investigating the upper limit of atmospheric erosion related to the LHB, we consider only the lowest limit of $n=10$ and examine the number of impactors which are necessary to erode the martian atmosphere since the late Noachian so that we end up with $\sim 7$ mbar.

If the martian atmosphere can be eroded by impacts only, the numbers of impacts above the critical mass has to be higher compared to the number computed in the exponentially decaying impact flux model. We found from our calculations that depending on the initially assumed surface pressure of $\sim 0.1-1$ bar, one would need $\sim 8000-15000$ impactors with masses equal or larger than $m_{\text {crit }}$ to erode the martian atmosphere to a surface pressure of $\sim 7$ mbars over the last 3.8 Gyr. However an exponentially decaying impactor flux model gives only $\sim 86$ for $\sim 0.1$ bar and $\sim 30$ for $\sim 1$ bar for the number of impacts above $m_{\text {crit }}$ over this time period. These numbers are $\sim 100-500$ times smaller than the necessary number of large impacts. Therefore, by considering an exponentially decaying impact flux, it is unlikely that the martian atmosphere with a surface pressure $\geq 0.1$ bar was eroded by impacts during the past $3.8 \mathrm{Gyr}$.

The LHB, on the other hand, can provide the number of large impactors which is necessary to remove the atmosphere from Mars. After a period which can most likely be characterized by a weak bombardment rate $~ 3.9$ Gyr ago, the planets experienced the LHB. The LHB was a cataclysmic episode characterized by a high bombardment rate, during a time-span of $\sim 50-300$ Myr. The Nice model (e.g., Gomes et al., 2005) which simulates the orbital evolution of the Solar System with slow migration of the giant outer planets, followed by a chaotic phase of orbital evolution, yields an estimate of impactor mass distribution during this period. The impactor masses could be distributed as presented in Fig. 11 during the late heavy bombardment period (data provided by Morbidelli, private communication). By investigating the best case for the erosion efficiency, we consider that the largest impactor provides the first impact.

The maximum diameter of the impactors hitting Mars as a function of time can be compared with the critical impactor diameter $d_{\text {crit }}$ that can erode the atmosphere. For an assumed initial surface pressure of $\sim 300$ mbar, the critical mass $m_{\text {crit }}$ and the corresponding critical impactor diameter $d_{\text {crit }}$ (with $\rho=2000 \mathrm{~kg}$ $\mathrm{m}^{-3}$ ) for different values of $n=m_{\text {crit }} / m_{\text {tan }}$ can be calculated. By assuming $n=10$, from these calculations one obtains an upper limit for the amount of atmosphere which can be eroded by impacts of $\sim 150$ mbar over an intense bombardment period of $\sim 0.3$ Gyr. Lower values of $n$ can erode primordial atmospheres of $\sim 400$ mbar $(n \approx 3)$ and even $\sim 1$ bar in the case of $n \sim 1)$. From these results one can see that impacts could have removed a major fraction of an accumulated secondary $\mathrm{CO}_{2}$ atmosphere (see Fig. 6). The main problem with the impact studies remains to be the choice of parameter $n$. As discussed in Sect. 4 studies which assume values for $n \geq 30$ deliver volatiles to the martian surface. Under this consideration, small $n$, and hence atmospheric erosion due to impacts as discussed before, is ques- 


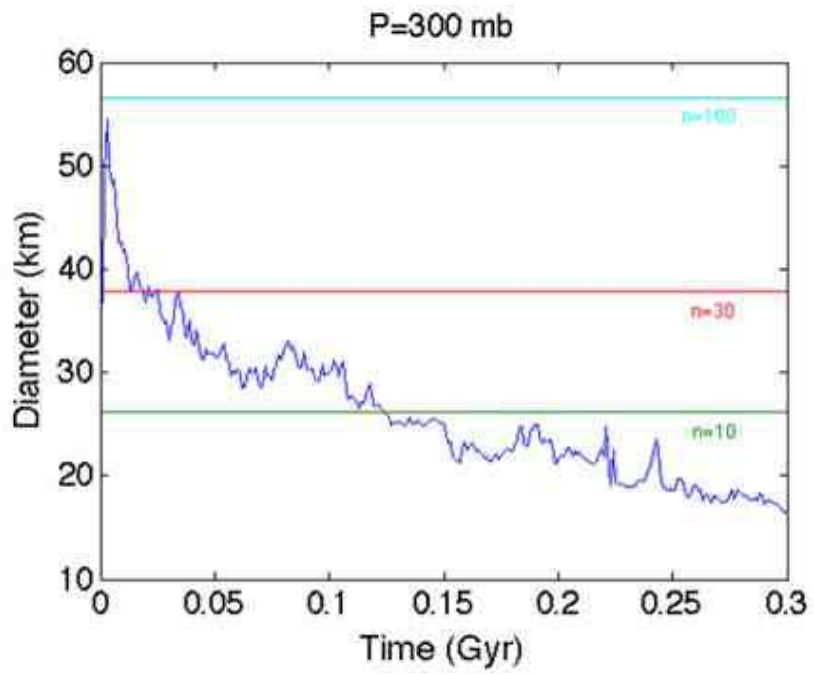

Fig. 11 Impactor diameters for the LHB for the best erosion case and for an initial pressure of $\sim 300$ mbar

tionable considering that recent hydrocode simulations suggest at least an order of magnitude larger value of $n$ (Svetsov 2007; Pham et al. 2009; 2010; 2011). In such a case the LHB would have accumulated volatiles additively to the secondary outgassed atmosphere. By assuming that delivered $\mathrm{CO}_{2}$ corresponds to $\sim 1 \%$ of the impactors this accumulation could result in an amount of impact delivered $\mathrm{CO}_{2}$ of $\sim 300$ mbar. Thus, the $\mathrm{H}_{2} \mathrm{O}$ which could have been brought to Mars by impacts (Levison et al. 2001) especially during the LHB-period, where the solar EUV flux and related thermal escape processes were much lower compared to their early values, could also be an important contribution to the planets present water inventory. The cometary bombardment, is largely unconstrained but can deliver up to or even more then $\sim 5$ bar of $\mathrm{H}_{2} \mathrm{O}$, that corresponds to a $\approx 130$ m deep GEL. These numbers should be considered as upper limits for the assumed total mass of the comets and asteroids $\left(7 \times 10^{22} \mathrm{~g}\right.$ and $4 \times 10^{22} \mathrm{~g}$, respectively) which may have fallen to Mars during the LHB (data provided by Morbidelli 2009, private communication).

Geomorphological and geological evidence shows that liquid water flowed on the martian surface, particularly in the Noachian period (Baker 2001; Squyres and Knoll 2005). In order to have liquid water stable on the martian surface, $\mathrm{CO}_{2}$ surface pressures of several bar are necessary to obtain temperatures above freezing (Kasting 1991). If one considers scattering of infrared radiation from $\mathrm{CO}_{2}$ ice clouds (Forget and Pierrehumbert 1997) or additional greenhouse gases such as $\mathrm{CH}_{4}, \mathrm{SO}_{2}$ and $\mathrm{H}_{2} \mathrm{~S}$, which could have also been released by volcanism (Kasting 1997; Johnson et al. 2008) this value can be achieved for $\sim 0.5-1.0$ bar. On the other hand aqueous solutions with lower melting points may have existed (e.g., Fairén, 2010, and references therein; Möhlmann 2012) making it possible that Mars might have been "cold-and-wet" with average surface temperatures of $\sim 245$ K (Fairén 2010; Gaidos and Marion 2003). Furthermore, water released by large 
impacts during the LHB could also have liberated huge amounts of water so that transient wet and warm conditions on the surface (Segura et al. 2002; Toon et al. 2010) could have occurred.

However, if impacts delivered volatiles additionally to the secondary atmosphere during the LHB period, this portion should have been lost partly to space during the Hesperian and Amazonian by various nonthermal atmospheric escape processes and partly weathered out of the atmosphere into the surface and ice.

\section{Escape and Surface Weathering of the Secondary Atmosphere Since the End of the Noachian}

From Mars Express ASPERA-3 ion escape data, Barabash et al. (2007) estimated the fraction of $\mathrm{CO}_{2}^{+}$molecular ions lost to space since the end of the Noachian when the martian dynamo stopped to work equivalent to a surface pressure of about $\sim 0.2-4$ mbar. The present $\mathrm{CO}_{2}^{+}$escape rates are about two orders of magnitude lower compared to the $\mathrm{O}$ loss and are on the order of $\sim 8 \times 10^{22} \mathrm{~s}^{-1}$ (Barabash et al. 2007).

That direct escape of $\mathrm{CO}_{2}^{+}$ions from Mars was low is also in agreement with various MHD and hybrid model results which yield an integrated $\mathrm{CO}_{2}^{+}$ion loss (IL) since the end of the Noachian on the order of $\sim 0.8-100$ mbar (e.g., Ma et al. 2004; Modolo et al. 2005; Chassefière and Leblanc 2007; Lammer et al. 2008; Manning et al. 2010). Moreover from a recent study of Ma et al. (2007) who calculated an escape rate of carbon of about 1.8 times larger at solar maximum than at solar minimum which is in good agreement with the dependency of the escape ion rate calculated in Fox et al. (2009) the estimated amount of $\mathrm{CO}_{2}$ lost by ion loss since $\sim 4$ Gyr is most likely not in excess of $\sim 1$ mbar (Chassefière and Leblanc 2011a). Thus, from these studies we can consider that the realistic $\mathrm{CO}_{2}^{+}$molecular ion loss by pick up and outflow through the martian tail in the theoretical range of about 0.8-100 mbar given in Manning et al. (2010) should be considered closer to the lower values.

On the other hand one should mention that all the previous ion escape models did not use an accurately modeled neutral atmosphere and ionosphere which corresponds to higher EUV fluxes expected before 2.5 Gyr ago. In such a case one can expect that more carbon dioxide will be dissociated in the thermosphere so that it can be heated to higher temperatures. As shown by Tian et al. (2009) a hotter thermosphere leads to an expansion of the upper atmosphere and thus more extended coronae. In such a case the solar wind interaction area would be larger and one may expect higher ion loss rates too.

One should also note that solar wind induced forcing of Mars can also result in outflow and escape of ionospheric ions. ASPERA-3 observations indicate that the replenishment of cold ionospheric ions starts in the dayside at low altitudes at $\sim 300-800 \mathrm{~km}$, where ions move at a low velocity of $\sim 5-10 \mathrm{~km} \mathrm{~s}^{-1}$ in the direction of the external magnetosheath flow (Lundin 2011). The dominating energization and outflow process, applicable for the inner magnetosphere of Mars, leads to outflow at energies of $\sim 5-20 \mathrm{eV}$. These energized "cool" ionospheric ions can be picked up, accelerated by the current sheet, by waves and parallel electric fields (Lundin 2011). The latter acceleration process can be observed above martian crustal magnetic field regions. But even if we assume that cold ionospheric ions 

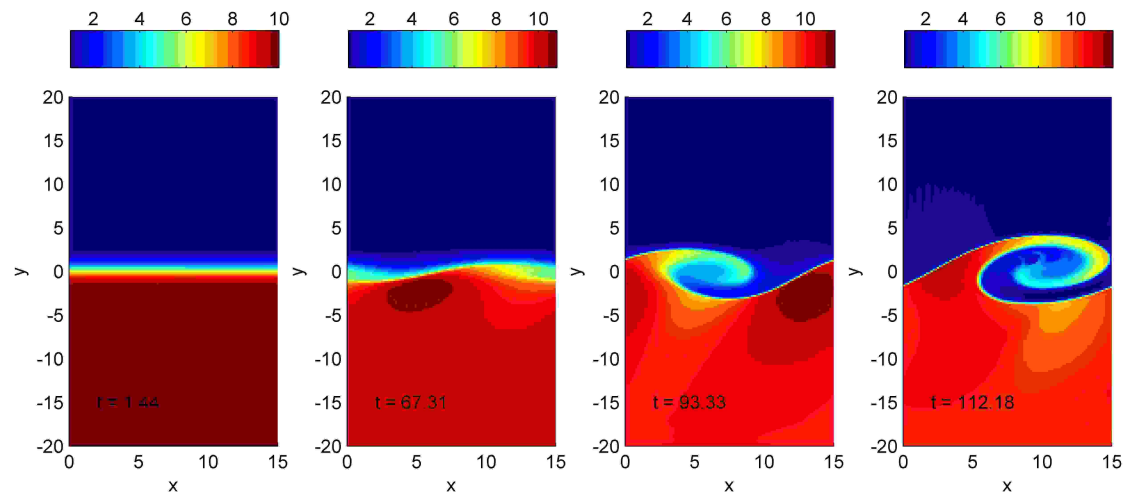

Fig. 12 Nonlinear evolution of the Kelvin-Helmholtz instability. The time series of the mass density is shown, from an MHD simulation with periodic boundary conditions in the $x$ direction. The mass density changes from the upper to the lower plasma layer and exhibits an increase of up to ten times (see the color code; blue: low density, red: high density). In the upper layer, the plasma flows from left to right. In the lower layer, the plasma is at rest. Initially small perturbations of the boundary layer separating the two plasma layers evolve into a $\mathrm{KH}$ vortex.

may enhance the ion escape for carbon bearing species the escape related to these processes most likely remains within the range given by Manning et al. (2010).

The Kelvin-Helmholtz (KH) plasma instability has also been regarded as a possible nonthermal atmospheric loss process around unmagnetized planets since Pioneer Venus Orbiter observed detached plasma structures, termed plasma clouds which contained ionospheric particles, downstream to the terminator in the magnetosheath of the planet (Brace et al. 1982; Wolff et al. 1980). Around planets, magnetopauses or ionopauses form boundaries with velocity shears, where the KH instability might be able to develop. On their way along the boundary from the subsolar point to the terminator, waves of initially small amplitudes grow and eventually form vortices in their nonlinear stage. When the vortex is able to detach, it carries ionospheric particles away and thus can contribute to the loss of ions (Brace et al. 1982).

Amerstorfer et al. (2010) and Möstl et al. (2011) performed recent numerical simulations of the $\mathrm{KH}$ instability with input parameters suitable for the boundary layers around unmagnetized planets. Fig. 12 shows a time series of the normalized mass density at different times during one of their simulations. After the linear growth time of the instability, a regular-structured vortex has evolved in the nonlinear stage. For this simulation, the density of the lower plasma layer is only ten times the density of the upper layer - a larger density jump stabilizes the boundary layer. The results of Möstl et al. (2011) indicates that the martian ionopause should be stable with regard to the KH instability due to the stabilizing effect of the large mass density of the ionosphere. However, the induced magnetopause (Venus) or magnetic pile-up boundary (Mars) might be $\mathrm{KH}$ unstable during high solar activity. For this boundary, the atmospheric loss of planetary ions might not be as severe as if the ionopause was the unstable boundary. Thus this recent result indicate that the loss due to the $\mathrm{KH}$ instability is not as significant as previously thought (Penz et al. 2004). Furthermore, at the altitudes where one can expect 
that under extreme solar conditions plasma clouds may detach from the upper atmosphere atomic oxygen ions should be the dominant species and $\mathrm{CO}_{2}^{+}$or $\mathrm{CO}^{+}$ ions are most likely negligible constituents.

Atmospheric sputtering (SP) has been identified as an escape process of heavy atoms from planetary bodies with low gravity such as Mars (Luhmann and Kozyra 1991). Leblanc and Johnson (2002) studied sputter escape of $\mathrm{CO}_{2}$ and $\mathrm{CO}$ from the martian atmosphere during the past $3.5 \mathrm{Gyr}$ with a coupled test particle Monte Carlo molecular dynamic model which considers collisions between photochemically produced suprathermal atoms and background molecules for EUV fluxes which are 3 times and 6 times higher than that of today's Sun. These authors obtained an escape of $\mathrm{CO}_{2}$ caused by sputtering since about 3.6-4 Gyr on the order of 50-60 mbar (see also Chassefière and Leblanc 2007). More recently, it has been argued that the flux of pick-up ions reimpacting Mars' atmosphere follows a logarithmic slope with the EUV flux of 1.8 (Chassefière and Leblanc 2011a), much flatter than the value of $\sim 8$ used in Chassefière et al. (2007), yielding a cumulated sputtering escape rate since $\sim 4$ Gyr which gives a $\mathrm{CO}_{2}$ loss $\leq 1$ mbar.

One can see that the sputter loss was probably similar to that of ion erosion and for sure not efficient enough to cause the loss of that hundreds of mbar of $\mathrm{CO}_{2}$ could be lost. Further, we note that the sputtering is a highly nonlinear process that depends on the EUV flux and the life time of the martian magnetic dynamo (Dehant et al. 2007). Furthermore, it was shown by Terada et al. (2009) that, due to the extreme solar wind atmosphere interaction caused by the young Sun before $\sim 4$ Gyr ago, a stronger induced magnetic field in the upper atmosphere could have decreased sputtering during the transition period when the planet's intrinsic dynamo stopped working.

Besides ion escape and sputtering the loss of exothermal photochemically produced suprathermal atoms such as $\mathrm{O}, \mathrm{C}, \mathrm{N}$ and $\mathrm{H}$ could have been more effective compared to both escape processes discussed before. Exothermal processes such as dissociative recombination (DR) of $\mathrm{O}_{2}^{+}, \mathrm{N}_{2}^{+}$or $\mathrm{CO}^{+}$ions produce neutral atoms in the ionosphere with higher kinetic energy compared to the background atmosphere (e.g., Ip 1988; Nagy et al. 1990; Kim et al. 1998; Lammer et al. 2000; Fox 2004; Fox and Hać 2009; Krestyanikova and Shematovich 2006; Chaufray et al. 2007; Valeille et al. 2009; Gröller et al. 2010; 2012). These newly created particles collide with the cooler background gas, lose energy by collisions, transfer energy so that a cold atom could become more energetic and finally a fraction of them reach the exobase level and if their energy is larger than the escape energy they are lost from the planet as neutrals. The production of these hot $\mathrm{O}$ and $\mathrm{C}$ atoms originating from $\mathrm{DR}$ of $\mathrm{O}_{2}^{+}$and $\mathrm{CO}^{+}$molecular ions is also strongly related to the solar EUV flux, and to an electron temperature dependent rate coefficient, where the total energy of these newly produced $\mathrm{O}\left({ }^{3} \mathrm{P},{ }^{1} \mathrm{D}\right), \mathrm{O}\left({ }^{3} \mathrm{P},{ }^{1} \mathrm{~S}\right)$ and $\mathrm{C}\left({ }^{3} \mathrm{P},{ }^{1} \mathrm{D}\right)$, $\mathrm{O}\left({ }^{3} \mathrm{P},{ }^{1} \mathrm{D},{ }^{1} \mathrm{~S}\right)$ atoms is a sum of their released energies $(\Delta E)$ according to a DR reaction channel of kinetic and internal energy, the latter being stored in molecules as vibrational and rotational energy.

Excited $\mathrm{C}$ atoms can also be produced via photo-dissociation $(\mathrm{PD})$ of $\mathrm{CO}$ molecules from $\mathrm{CO}+\mathrm{h} \nu \rightarrow \mathrm{C}\left({ }^{3} \mathrm{P}\right)+\mathrm{O}\left({ }^{3} \mathrm{P}\right)+\Delta \mathrm{E}$, with $\Delta E$ obtained as the difference of the photon energy and the energy which is needed to dissociate the molecule and excite the newly produced atoms. Fox (2004) made a complete calculation of all possible photochemical channels for the production of carbon escaping particles and found that between $7.5 \times 10^{23} \mathrm{C} \mathrm{cm}^{-2} \mathrm{~s}^{-1}$ to $\sim 4.5 \times 10^{24} \mathrm{C} \mathrm{cm}^{-2} \mathrm{~s}^{-1}$ may 
Table 4 Estimated min. and max. outgassed (VO: volcanic outgassing) $\mathrm{CO}_{2}$ in units of bar $\sim 4$ Gyr ago and the expected min. and max. range of impact eroded (IE) or delivered (ID) atmosphere during the late heavy bombardment ( $\mathrm{LHB}$ ) period. $\mathrm{CO}_{2}$ escape of various atmospheric loss processes (IL: ion loss; KH: Kelvin Helmholtz instability triggered ionospheric detached plasma clouds; SP: sputtering; DR: dissociative recombination; PD: photo dissociation) from observations and models integrated since that time.

\begin{tabular}{l|c}
\hline Sources and loss processes & $\mathrm{CO}_{2}[\mathrm{bar}]$ \\
\hline VO $\sim 4$ Gyr ago, max. & $\sim 0.2-0.5 \mathrm{bar}$ \\
VO $\sim 4$ Gyr ago, min. & $\sim 0.05$ bar \\
\hline ID $\sim$ LHB & $\sim 0-0.3$ bar \\
IE $\sim$ LHB & $\sim 0-0.15$ bar \\
\hline IL since $\sim 4$ Gyr ago & $\sim 001-0.1$ bar \\
KH since $\sim 4$ Gyr ago & $\leq 0.001-0.05$ bar \\
SP since $\sim 4$ Gyr ago & $\sim 0.001-0.1$ bar \\
DR since $\sim 4$ Gyr ago & $\leq 0.005-0.05$ bar \\
PD since $\sim 4$ Gyr ago &
\end{tabular}

escape from solar minimum to maximum conditions with photo-dissociation of $\mathrm{CO}$ being the most efficient process.

If we apply a recently developed hot atom Monte Carlo model which selects the magnitude of the initial velocity of a newly produced hot particle randomly from the calculated velocity distribution, which considers collisions that are based on the energy dependent total and differential cross sections for elastic, inelastic and quenching collisions and numerous cascaded hot particles (Gröller et al. 2010; 2012) to present martian conditions, we obtain total escape rates for "hot" O atoms of $\sim 9 \times 10^{25} \mathrm{~s}^{-1}$ and for hot $\mathrm{C}$ atoms $\sim 2.7 \times 10^{25} \mathrm{~s}^{-1}$ during high solar activity conditions and $\sim 3 \times 10^{25} \mathrm{~s}^{-1}$ for "hot" $\mathrm{O}$ atoms and $\sim 3 \times 10^{24} \mathrm{~s}^{-1}$ for hot $\mathrm{C}$ atoms during low solar wind conditions. Our obtained hot $\mathrm{O}$ escape rates from present Mars are in good agreement with Chaufray et al. (2007) and Valeille et al. (2009) and about a factor of 5 to 3 lower compared to results from Fox and Hać (2009) for high and low solar activity, respectively.

If we apply our model to the thermosphere/ionosphere profiles modeled by Tian et al. (2009) during the earlier periods of martian history preliminary estimates yield an integrated total $\mathrm{CO}_{2}$ loss from today to 4 Gyr ago of $\leq 100$ mbar. A smaller value of $\leq 10$ mbar has been proposed by Chassefière and Leblanc (2011a).

Table 5 compares expected max. and min. outgassed and accumulated $\mathrm{CO}_{2}$ atmosphere in units of bar at $\sim 4$ Gyr and the integrated min. and max. $\mathrm{CO}_{2}$ escape of various nonthermal atmospheric loss processes from observations and models since that time. Table 5 shows a very large dispersion of one or two orders of magnitude yielding a range of total $\mathrm{CO}_{2}$ loss from less than $\sim 10$ mbar up to $\sim 100$ mbar, which is definitely smaller than the expected upper values of an outgassed and accumulated secondary $\mathrm{CO}_{2}$ atmosphere. However, a similar amount of $\mathrm{CO}_{2}$ or even a higher one could be stored in the surface sinks which we discuss in the next section. 


\section{Surface Sinks of $\mathrm{CO}_{2}$ and $\mathrm{H}_{2} \mathrm{O}$}

Estimates of the total amount of $\mathrm{CO}_{2}$ degassed into the martian atmosphere since accretion vary between $\sim 5-12$ bar, while as shown in Sect. 5 most of it was lost to space during the period of the young and active Sun. However, most of the strong EUV-powered atmospheric loss occurred most likely prior or during the early period of the Noachian and the Hellas impact ( $\sim 4.0 \mathrm{Gyr})$. As it was discussed in the previous Sections the lost amount to space of $\mathrm{CO}_{2}$ which accumulated from volcanic outgassing and/or was delivered by impacts during the LHB, is most likely not higher than $\approx 100$ mbar. Therefore, if Mars had indeed a denser $\mathrm{CO}_{2}$ atmosphere $~ 3.5-4$ Gyr ago, most of it should be hidden below the planet's surface.

\subsection{Sequestration of $\mathrm{CO}_{2}$ in Carbonates}

Possible sinks for this $\mathrm{CO}_{2}$ atmosphere include loss to space, adsorption on the regolith, deposits of $\mathrm{CO}_{2}$-ice, and deposits of carbonate minerals. The maximum $\mathrm{CO}_{2}$ adsorbed on the regolith has been estimated to be on the order of $\sim 30-40$ mbar and is likely to be less than that (Zent and Quinn 1995). Recent discoveries of buried $\mathrm{CO}_{2}$-ice deposits in the polar regions indicate that modern $\mathrm{CO}_{2}$-ice deposits could approach $\sim 20-30$ mbar equivalent $\mathrm{CO}_{2}$ (Phillips et al. 2011). Combining these sinks with the amount of $\mathrm{CO}_{2}$ likely lost to space since the past $\sim 4$ Gyr (see Table 5), it becomes clear that the majority of $\mathrm{CO}_{2}$ that could be accounted for is most likely $<150$ mbar.

Therefore, the amount of $\mathrm{CO}_{2}$ which is possibly stored as carbonate becomes the key to understanding the density of the ancient martian atmosphere at the end of the Noachian since it is the only sink that can accommodate a dense Noachian atmosphere. Early studies modeling a dense atmosphere on Mars predicted abundant carbonates in the martian crust (Pollack et al. 1987), but after a decade of intense exploration of Mars from orbit and on the surface, abundant carbonate deposits have not been discovered (e.g., Bibring et al. 2007). However, the detections of carbonates that have been made to date have revealed deposits that are either buried or widely dispersed making detection difficult (Ehlmann et al. 2008; Michalski and Niles 2010; Morris et al. 2010). Therefore, orbital detection of carbonates may not provide a complete view of the carbonate crustal reservoir on Mars which may be larger than currently expected. Nevertheless, information from martian meteorites, landers, rovers, and orbiters is now available to construct a fairly consistent story of the carbonate reservoir on Mars.

The most direct evidence for carbonates on Mars comes from martian meteorites, many of which contain carbonate minerals in trace abundances $(<1 \%)$ (Bridges et al. 2001). There are currently 40-50 known meteorites which are derived from 3-6 distinct sites on Mars (Eugster et al. 2002). These rocks are derived from energetic ejection events from the martian surface which likely destroyed all but the strongest igneous rocks providing a selection bias. Therefore it is clear that while martian meteorites are invaluable samples of the martian surface, they likely do not provide an adequate sample by which to judge the carbonate crustal reservoir of Mars. They do indicate that weathering and carbonate formation on Mars has been active at least at very low levels throughout the Noachian, Hesperian, and Amazonian periods (Gooding et al. 1988; Mittlefehldt 1994; Bridges et al. 2001; 


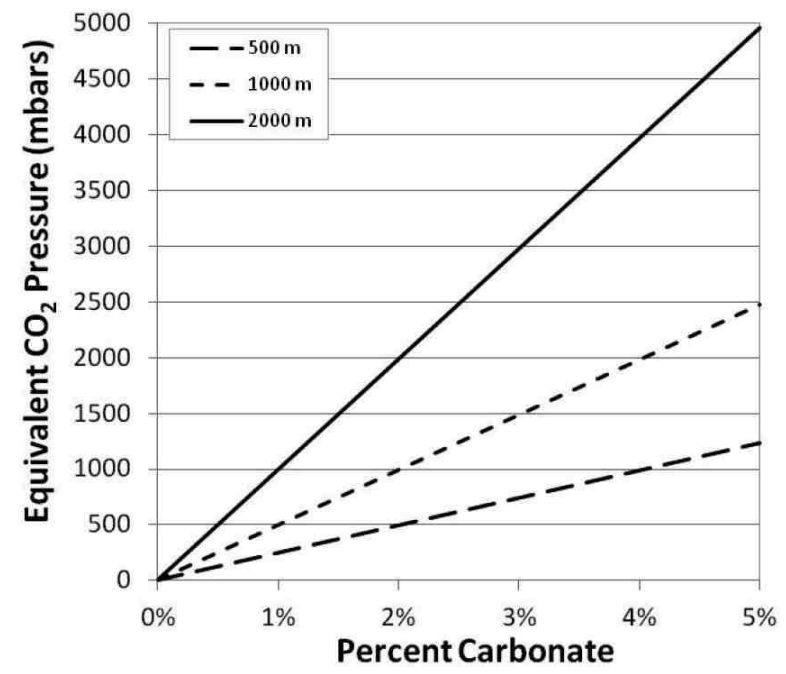

Fig. 13 Calculation of equivalent $\mathrm{CO}_{2}$ atmospheric pressures based on carbonate reservoir size calculated from volume percentage of carbonate and depth of carbonate mineralization.

Niles et al. 2010). Localized deposits of more concentrated carbonates have also been found in some of the oldest Noachian terrains on Mars. These deposits, which have mostly been identified spectroscopically from orbit, are typically mixtures of carbonate minerals with other phases including serpentine, olivine, smectite clays, and pyroxene minerals (Ehlmann et al. 2008; Michalski and Niles 2010; Morris et al. 2010). They are also buried underneath younger volcanic or ejecta deposits indicating ages that likely date to the early to mid Noachian.

The carbonate abundance in these deposits ranges from $~ 10-30 \%$ (Ehlmann et al. 2008; Michalski and Niles 2010; Morris et al. 2010). In Gusev crater, a carbonate deposit was identified by the Spirit rover using spectroscopic, chemical, and Mössbauer data. This deposit is also consisted of carbonate mixed with volcanoclastic materials, with carbonate abundances of $\sim 16-34 \%$. Carbonates have also been detected in the dust and soils of Mars to be present at abundances between $\sim 2-5 \%$ (Bandfield et al. 2003). This is perhaps our best means for estimating the crustal reservoir of carbonate on Mars as the dust on Mars is globally mixed (McSween and Keil 2000) and may be representative of the average composition of the upper crust of the planet. However, the dust is very fine grained and might be highly susceptible to weathering under current martian conditions (Shaheen et al. 2010), and so might reflect an artificially elevated carbonate abundance due to atmospheric or near surface weathering. Nevertheless, the carbonate content of the dust can provide at least a hypothetical upper boundary for the carbonate content of the crust.

Fig. 13 shows the equivalent atmospheric pressure for different crustal abundances of carbonate, assuming $5 \%$ as an upper boundary in the crust and crustal thickness up to $2 \mathrm{~km}$. As one can see, a maximum amount of $\mathrm{CO}_{2}$ which could hypothetically be stored in the crust could be up to $\sim 5$ bar with the assumption that the dust is a representative sample of the top $2 \mathrm{~km}$. Of course this estimate 
is for the total $\mathrm{CO}_{2}$ reservoir in the martian crust, and only part of this or even a tiny fraction of a carbonate reservoir formed since the Noachian.

It is difficult to determine how much of this carbonate reservoir can be attributed to the $\mathrm{CO}_{2}$ presence during the Noachian when many of the valley networks and phyllosilicates formed, but EUV-powered atmospheric escape was high. The assumption that not much carbonates may have formed after the early Noachian is in agreement with the nature of the carbonate deposits which have been discovered so far, as they all are dated at the early to mid-Noachian or earlier (Ehlmann et al. 2008; Michalski and Niles 2010; Morris et al. 2010).

Major outstanding issues remain in our understanding of the carbonate reservoir on Mars including:

- What was and is the total carbonate reservoir on Mars and how much carbonates are stored in the deep crust $(>5 \mathrm{~km})$ ?

- How efficient were environmental processes, which acted against carbonate formation?

- How much carbonate has formed since the start of the Noachian and how is it distributed through time?

- How efficiently are carbonates recycled back into the atmosphere on Mars and through what mechanisms?

\section{2 $\mathrm{CH}_{4}$ as a Clue to an Active Long-Term Carbon Cycle}

During the last decade, $\mathrm{CH}_{4}$ has been detected in Mars atmosphere by different instruments from both Earth and spacecraft in orbit around Mars (Planetary Fourier Spectrometer (PFS) on Mars Express, Thermal Emission Spectrometer (TES) on Mars Global Surveyor (MGS)) at an average 10-20 ppb level (Krasnopolsky et al. 2004; Formisano et al. 2004; Mumma et al. 2009; Fonti and Marzo 2010). One of the most striking characteristics of the observed $\mathrm{CH}_{4}$ is its high temporal and spatial variability, implying a lifetime of $\sim 200$ days (Lefèvre and Forget 2009), much shorter than the currently admitted value of $\sim 300 \mathrm{yr}$ based on existing photochemical models (e.g. Krasnopolsky 2006). Such a large discrepancy, together with the small signal to noise ratio of spacecraft data, led some scientists to consider the detection of $\mathrm{CH}_{4}$ as questionable. Concerning data acquired from Earth (Mumma et al. 2009), it has been argued that the coincidence between martian and telluric lines could have led to an erroneous retrieval of the $\mathrm{Mars} \mathrm{CH}_{4}$ abundance (Zahnle et al. 2011). Nevertheless, spacecraft data from MGS and Mars Express which now span $~ 6$ martian years (MY24-MY29) and Earth-based measurements show a globally consistent and reproducible seasonal behaviour of the $\mathrm{CH}_{4}$ mixing ratio with similar abundance levels and amplitudes of variation (see Fig. 1 in Chassefière and Leblanc 2011b; Mousis et al. 2012, this issue), reasonably suggesting that $\mathrm{CH}_{4}$ is effectively present in the martian atmosphere. All existing observations show that $\mathrm{CH}_{4}$ concentrations are spatially and temporally highly variable, and its lifetime has to be on the order of $\sim 90$ days. $\mathrm{CH}_{4}$ could be oxidized in the superficial regolith layer through heterogeneous chemistry processes involving gaseous oxidants like $\mathrm{H}_{2} \mathrm{O}_{2}$ and/or direct oxidation by ferric iron at the surface of grains (Chassefière and Leblanc 2011a).

The calculated present $\mathrm{CH}_{4}$ release flux, as deduced from present abundance measurements ( $\sim 10$ ppbv $\mathrm{CH}_{4}$ in a seasonal average, Geminale et al. 2008; 2011; 
Fonti and Marzo 2010) and estimates of the $\mathrm{CH}_{4}$ lifetime (200 days, Lefèvre and Forget, 2009) is on the order of $\sim 1.0 \times 10^{8} \mathrm{~cm}^{-2} \mathrm{~s}^{-1}$. Such a high flux is difficult to explain by an external (meteoritic) source and direct volcanism releasing only trace amounts of reduced carbon can be similarly ruled out as a major source of $\mathrm{CH}_{4}$ (Atreya et al. 2006). According to these authors, hydrothermalism and/or biological activity may be at the origin of $\mathrm{CH}_{4}$ on Mars. It has been suggested by Dohm et al. (2008) that Mars is still internally active, with a potential for continuing magmatic-driven activity (Hauber et al. 2011), including volcanism and hydrothermal activity, supporting a possible hydrothermal origin.

Hydrothermalism may result in the production of $\mathrm{CH}_{4}$, either by reduction of $\mathrm{CO}_{2}$ through water-rock interaction at low oxygen fugacity (Lyons et al. 2006), or through serpentinization followed by the conversion of $\mathrm{H}_{2}$ in $\mathrm{CH}_{4}$ (Oze and Sharma 2005). Whatever the origin of $\mathrm{CH}_{4}$, assumed to take place in the crust below the water table, the fate of the produced $\mathrm{CH}_{4}$ is to be transported upward by ascending hydrothermal fluids and to be stored in the cryosphere in the form of $\mathrm{CH}_{4}$ clathrate hydrates (Chassefière and Leblanc 2011a; Mousis et al. 2012; this issue).

For how long has $\mathrm{CH}_{4}$ been released at the present rate to the atmosphere? A few arguments in favour of a long-term and relatively continuous phenomenon have been proposed:

- If $\mathrm{CH}_{4}$ oxidation is at the origin of the suspected present redox imbalance between the $\mathrm{H}$ and $\mathrm{O}$ escape fluxes, the release rate of methane averaged over the last $10^{3} \mathrm{yr}$ (photochemical lifetime of $\mathrm{H}_{2}$ ) required to explain the presumably small $\mathrm{O}$ escape rate, is close to the present release rate (Chassefière and Leblanc 2011b). This suggests that methane could have been released at an average release rate similar to the present one for at least a few thousand years.

- As pointed out by Chassefière and Leblanc (2011b; 2011c), the quantity of carbon contained in the superficial layer of $\mathrm{CO}_{2}$-ice covering the permanent south polar cap is comparable to the amount of $\mathrm{CH}_{4}$ which would be delivered to the atmosphere, then converted to $\mathrm{CO}_{2}$, over a time interval of $3 \mathrm{Myr}$, that is the time since the last obliquity transition period (Levrard et al. 2004). This small amount of $\mathrm{CO}_{2}, \sim 1 \%$ of the atmospheric mass, would be at condensation equilibrium with larger amounts of $\mathrm{CO}_{2}$-ice sequestered in the martian south polar layered deposits, recently discovered by the Shallow Subsurface Radar (SHARAD) on Mars Reconnaissance Orbiter (MRO) (Phillips et al. 2010). The thin superficial $\mathrm{CO}_{2}$-ice layer covering the south polar cap could therefore originate in the hydrothermal $\mathrm{CH}_{4}$, as well as possibly some volcanic $\mathrm{CO}_{2}$, released since the time of the transition. This suggests a $\mathrm{CH}_{4}$ release rate similar to the present value over the last few million or ten million years.

- If, as may seem likely, $\mathrm{CH}_{4}$ is released to the atmosphere from a $\mathrm{CH}_{4}$ clathraterich cryosphere, the release rate of $\mathrm{CH}_{4}$, produced sporadically at depth by hydrothermal activity, is considerably smoothed over a time scale of the order of the lifetime of $\mathrm{CH}_{4}$ clathrate, that is the time for the cryosphere to fully sublimate to the atmosphere and possibly be recycled to the crust, which may have occurred in the past. This time is estimated to be or the order of $\sim 10^{8}$ $10^{9}$ yr (Mousis et al. 2012, this issue), suggesting that $\mathrm{CH}_{4}$ could have been continuously released to the atmosphere over geological time scales. 
By scaling the $\mathrm{CH}_{4}$ release rate on the level of hydrothermal activity, assumed to be proportional to the lava extrusion rate as estimated from the geomorphological analysis of the surface (Greeley and Schneid 1991; Craddock and Greeley 2009), a cumulated amount of $\mathrm{CO}_{2}$ resulting from $\mathrm{CH}_{4}$ release since the Noachian of $\sim 2$ bar has been estimated (Chassefière and Leblanc 2011a). Assuming that $\mathrm{CH}_{4}$ release is due to serpentinization, a released amount larger than $\sim 0.4$ bar is not consistent with the present D/H ratio (see Sect. 3.1) in the martian atmosphere (Chassefière and Leblanc, 2011c). Up to $\sim 0.4$ bar of $\mathrm{CO}_{2}$ could therefore have been released in the form of $\mathrm{CH}_{4}$, then oxidized. It should be noted that $\mathrm{CH}_{4}$ outgassing is not an additional source of carbon with respect to the $\mathrm{CO}_{2}$ volcanic source described in the previous section. Either magmatic $\mathrm{CO}_{2}$ is converted to $\mathrm{CH}_{4}$ before being released to the atmosphere through fluid-rock interaction in deep hydrothermal fluids (Lyons et al., 2006), or it precipitates in crustal carbonates, with further hydrothermal decomposition to $\mathrm{CO}_{2}$, reduced to $\mathrm{CH}_{4}$ by the molecular hydrogen produced by serpentinization (Oze and Sharma 2005) and/or direct thermodynamical equilibration (Lyons et al. 2006). Subsurface hydrothermal activity could be responsible for both the release of $\mathrm{CH}_{4}$ to the atmosphere, and the recycling of atmospheric $\mathrm{CO}_{2}$ to the crust with further precipitation of carbonates, recycled later to the atmosphere under reduced form through hydrogeochemical processes (Chassefière and Leblanc 2011a).

Carbonate mineral deposits may occur in subsurface hydrothermal systems from liquid water, rich in dissolved $\mathrm{CO}_{2}$. Provided relevant subsurface zones are not entirely sealed from the atmosphere, some $\mathrm{CO}_{2}$ can be transferred from the atmosphere to subsurface water reservoirs and further precipitate in carbonates, as observed on Earth and expected to occur on Mars from geochemical modelling (Griffith and Shock 1995). Such a long term carbon cycle, with a progressive net removal of $\mathrm{CO}_{2}$ from the atmosphere and subsequent carbonate deposition in the subsurface, could explain the disappearance of a possible Noachian $\mathrm{CO}_{2}$ atmosphere built by volcanism and late impacts.

Because a carbon atom may have been cycled several times through the crust since its release from the mantle by volcanism, a cumulated $\mathrm{CH}_{4}$ release rate up to $\sim 0.4$ bar should not be interpreted as the content of a subsurface isolated reservoir. It rather suggests that an efficient carbon cycle has been maintained by hydrothermal processes, probably until the early Amazonian and possibly the present epoch, with a substantial fraction of the volcanic outgassed carbon being cycled one or several times through crustal carbonates.

\subsection{Storage Capability of $\mathrm{H}_{2} \mathrm{O}$ in the Martian Crust}

The present inventory of water on Mars is poorly constrained. The total water content of the two perennial polar caps corresponds to a EGL of $\mathrm{H}_{2} \mathrm{O}$ of $\sim 16$ $\mathrm{m}$ depth (Smith et al. 2001), and the ice deposits sequestered in the Dorsa Argentea Formation (DAF), near the south polar cap, could have represented $\sim 15$ $\mathrm{m}$ in the past (Head and Pratt 2001). Nevertheless, only a fraction of the initial water could remain today in the DAF reservoir, corresponding to $\sim 5-7.5 \mathrm{~m}$. Other reservoirs, expected to have been active during late Amazonian, could be present in tropical and mid-latitude regions (e.g., Watters et al. 2007; Holt et al. 2008; Head and Marchant 2009), but they probably represent only a minor 
contribution to the global reservoir. The total inventory of the known reservoir, including near-surface repositories that are distributed across middle to high latitudes, has been estimated to correspond to a 35 m thick EGL (Christensen 2006). The megaregolith capacity is large, with up to $\sim 500$ m hypothetically trapped in the cryosphere, and hypothetically several additional hundreds of meters (up to $\sim 500 \mathrm{~m}$ ) of ground water surviving at depth below the cryosphere (Clifford et al. 2010). It has been suggested that most of ground ice has been lost by sublimation at low latitudes, and that only small amounts of ground water would survive today (Grimm and Painter, 2009), with therefore less water in the megaregolith. Carr (1987) suggested that a $\sim 500 \mathrm{~m}$ thick EGL of water has to be required to explain the formation of outflow channels and most of this $\mathrm{H}_{2} \mathrm{O}$ could be trapped today as water ice, and possibly deep liquid water, in the subsurface.

Some of the water present on Mars may have reacted with minerals to form clay minerals and sulfates (Bibring et al. 2006; Mustard et al. 2008; Ehlmann et al. 2012 , this issue). The presence of these hydrated minerals at the surface of Mars suggests that hydration processes have been active on Mars in the past. They may have been formed, either at the surface of Mars during the Noachian, when liquid water was flowing at the surface of the planet, or in the subsurface by aqueous alteration of subsurface rocks, and possibly by impacts able to provide subsurface water to the impacted material (Bibring et al. 2006). Existing geochemical model calculations show that hydrothermal hydration of martian crust is an efficient process (Griffith and Shock 1997). Calculations have been made for a temperature in the range from $150-250^{\circ} \mathrm{C}$. The results do not depend much on the oxygen fugacity, and are similar for a moderately oxidized crust and for a highly oxidized medium. The final conclusion of this study is that water storage via hydrous minerals can account for $\sim 5$ wt $\%$ of crustal rocks. The capacity of the upper $10 \mathrm{~km}$ of the crust in storing water in the form of hydrated minerals therefore hypothetically corresponds to a few hundreds meter depth EGL of $\mathrm{H}_{2} \mathrm{O}$, but the crustal content of hydrated minerals is basically unknown and may be much lower than this upper limit.

The effectiveness and amplitude of aqueous alteration processes in Mars' crust are basically unknown. SNC meteorites, originating in martian crust and mantle, provide information on crustal geochemical processes. Formed of mantle material modified through interaction with crustal material during the upward migration of lava through the crust, they have been shown to record a wide range of oxidation conditions (Wadhwa 2001). Shergottites present a range of redox conditions from close to the IW-buffer up to that of the quartz-fayalite-magnetite buffer, which can be interpreted as the result of oxidation of the crust by a process such as aqueous alteration, through the oxidation of ferrous iron into ferric iron. Whereas Nakhlites and Chassignites are oxidized, the ancient ALH 84001 is relatively reduced. These results suggest that the silicates of SNC meteorites originate in a water-depleted martian mantle. These silicates would have been oxidized through assimilation of oxidized crustal material. In order to produce such an oxidation, a significant proportion of crustal material (10-30\%) with a high $\mathrm{Fe}_{3}+/ \mathrm{Fe}$ ratio $(\sim 50 \%)$ must have been mixed with mantle material (Herd et al. 2002). ALH84001, because it has crystallized at the end of the Noachian $~ 3.9$ Gyr ago, could have not been mixed with oxidized crust material.

The occurrence in the crust of aqueous alteration processes, as proved by oxidation processes recorded in SNC meteorites, suggests that the conditions for an 
efficient hydration of crustal rocks may have been met at some places and times in the past. Such hydrated minerals have been found in Nakhlites. A particular hydration process occurring in Earth's crust is serpentinization, which generates $\mathrm{H}_{2}$ from the reaction of water with ferrous iron derived from minerals, primarily olivine and pyroxene (McCollom and Back 2009). In the reaction, ferrous iron is oxidized by the water to ferric iron, which typically precipitates as magnetite, while hydrogen from water is reduced to $\mathrm{H}_{2}$. Iron oxidation is accompanied by the storage of a large number of water molecules in serpentine, an hydrated mineral which has been recently observed by the Compact Reconnaissance Imaging Spectrometer for Mars (CRISM) on MRO in and around the Nili Fossae region (Ehlmann et al. 2009). Serpentinization occurring in crustal hydrothermal systems is a plausible process at the origin of the methane observed in the martian atmosphere (Oze and Sharma 2007). Based on an analysis of the present Mars' D/H ratio, it has been suggested that a water GEL of up to 300-400 m depth could have been stored in crustal serpentine since the late Noachian due to hydrothermalism triggered by magmatic activity (Chassefière and Leblanc 2011). Massive serpentinization of the southern crust could have been at the origin of both the crustal dichotomy and the strong remanent magnetic field of old southern terrains (Quesnel et al. 2009).

Although there is no direct observational evidence of active hydration processes in Mars' crust, aqueous alteration processes, e.g. serpentinization, are potentially able to store several hundreds of meters of $\mathrm{H}_{2} \mathrm{O}$ in crustal hydrates. This amount is comparable to the estimated value of the water ice content of the cryosphere. Depending on the efficiencies of the various atmospheric escape processes and volatile delivery by impacts, several hundreds of meters of water could hypothetically be trapped in the subsurface in the form of $\mathrm{H}_{2} \mathrm{O}$-ice and/or hydrated minerals, and possibly liquid water below the cryosphere. All these reservoirs could exchange with each other, as well as with the atmosphere and polar caps (Lasue et al. 2012), at the occasion of magmatic and hydrothermal events, and could contain hypothetically up to $1 \mathrm{~km}$ thick EGL of water.

\section{Conclusions}

The latest hypotheses on the formation, outgassing and evolution of the martian atmosphere from the early Noachian up to the present time as illustrated in Fig. 14 have been discussed. Depending on the captured nebula gas and/or outgassed amounts of volatiles, we show that due to the high EUV flux of the young Sun the planet's hydrogen-rich protoatmosphere was lost via hydrodynamic escape of atomic hydrogen which could have dragged heavier atoms such as $\mathrm{C}$ and $\mathrm{O}$ during the first tens or hundreds of Myr after Mars' finished its accretion. The early Noachian impacts may have kept the protoatmosphere in vapor form and may not have much contributed to atmospheric growth because the delivered volatiles would have also escaped. After Mars lost its protoatmosphere the atmospheric escape rates were most likely balanced with a secondary outgassed atmosphere and delivered volatiles by impacts until the activity of the young Sun decreased so that the atmospheric sources could dominate over the losses. Depending on assumptions related to geochemical conditions such as the $\mathrm{pH}$ of the early martian environment, the global melt channel, melting in mantle plumes, oxygen fugacities and IWbuffers in combination with atmospheric escape and impact delivery during the late 


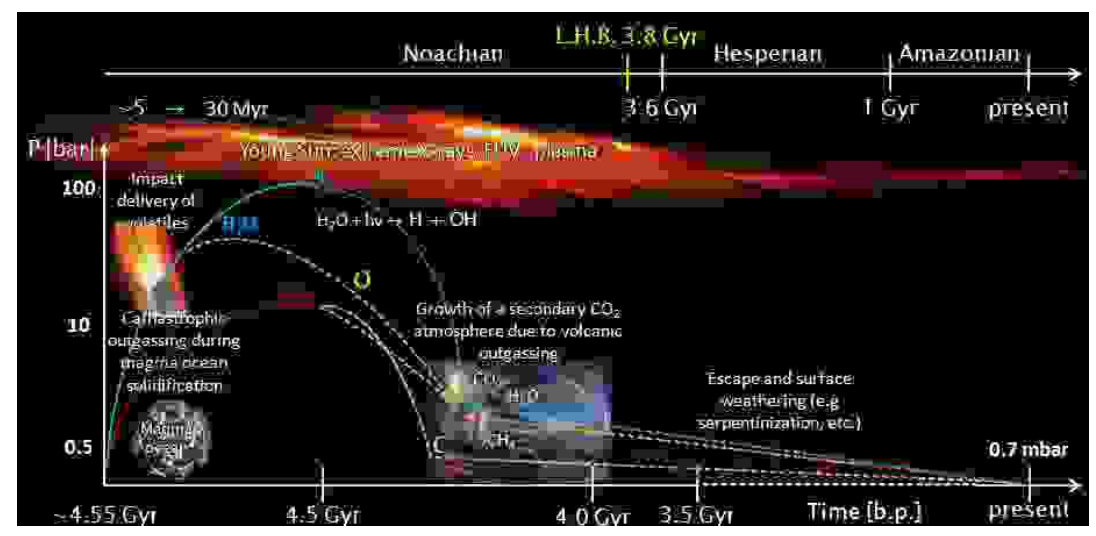

Fig. 14 Illustration of Mars' atmosphere evolution after the outgassing of volatiles and fast growth of a dense water vapour dominated $\mathrm{H}_{2} \mathrm{O} / \mathrm{CO}_{2}$ atmosphere during and after the magma ocean solidification process. blue lines: water and hydrogen; yellow lines: oxygen; red lines $\mathrm{CO}_{2}$. Solid lines: Outgassed or delivered species; dashed-lines: escaping species. A complex interplay between the young Sun's EUV activity, impacts and atmospheric escape processes during the first 500 Myr kept early Mars most of the time cool and dry. After the EUV flux of the young Sun decreased at $\sim 4-4.3$ Gyr ago, volcanic outgassing and impacts during the LHB could have resulted in the build-up of a denser and slightly warmer and wetter $\mathrm{CO}_{2}$ atmosphere of $\leq 1$ bar. If the secondary $\mathrm{CO}_{2}$ atmosphere after the Noachian had a surface pressure which was larger than $\sim 100$ mbar, only a minor fraction escaped to space during the Hesperian and Amazonian and most of it should be stored so far undiscovered in the subsurface. $\mathrm{CO}_{2}$ can be released from surface reservoirs during periods of climate change or impacts (non-linear dashed red line from the late Noachian until present) and may have modified the atmospheric surface pressure several times during the planet's history.

heavy bombardment Mars may have built up a secondary $\mathrm{CO}_{2}$ atmosphere which was $<1$ bar and accumulated a water inventory equivalent to $<10$ bar at $\sim 3.5-4$ Gyr ago. By reviewing the latest observations and model studies on the escape of the martian $\mathrm{CO}_{2}$ atmosphere during the Hesperian and Amazonian epochs we expect that the planet may have lost most likely less than $\sim 150$ mbar of $\mathrm{CO}_{2}$. If a $\mathrm{CO}_{2}$ atmosphere of several 100 mbar was indeed present at the end of the Noachian, most of it should have been removed to the crust by sequestration in carbonate rocks and partially recycled to the atmosphere under reduced and/or oxidized form. The water contained in a several $10 \mathrm{~m}$ or even $100 \mathrm{~m}$ deep EGL at the Noachian could have been trapped hypothetically in the crust in the form of $\mathrm{H}_{2} \mathrm{O}$-ice, hydrated minerals, and possibly liquid water under the cryosphere. On the other hand, if there are not enough hidden $\mathrm{CO}_{2}$ deposits under the planet's surface, then other greenhouse gases are necessary for the explanation of standing bodies of liquid water on the planet's surface $\geq 3.5-4$ Gyr ago.

Acknowledgements D. Breuer, E. Chassefière, M. Grott, H. Gröller, E. Hauber, H. Lammer, P. Odert and A. Morschhauser acknowledges support from the Helmholtz Alliance project "Planetary Evolution and Life". E. Chassefière acknowledges support from CNRS EPOV interdisciplinary program. H. Lammer acknowledge the support by the FWF NFN project S116 "Pathways to Habitability: From Disks to Active Stars, Planets and Life", and the related FWF NFN subproject, S116607-N16 "Particle/Radiative Interactions with Upper Atmospheres of Planetary Bodies Under Extreme Stellar Conditions". H. Gröller and H. Lammer acknowl- 
edges also support from the Austrian FWF project P24247-N16 "Modelling of non-thermal processes in early upper atomospheres exposed to extreme young Sun conditions" and support from the joined Russian-Austrian project under the RFBR grant 09-02-91002-215-ANF-a and the Austrian Science Fund (FWF) grant I199-N16. P. Odert was supported via the FWF project grant P19446-N16 and the research by U. Möstl was funded by the FWF project grant P21051-N16. O. Karatekin thanks A. Morbidelli for the discussions related to impact studies and the LHB; O. Karatekin, V. Dehant and L. B. S. Pham acknowledges the support of Belgian PRODEX program managed by the ESA in collaboration with the BELSPO. O. Mousis acknowledges support from CNES. P. Niles acknowledges support from NASA Johnson Space Center and the Mars Fundamental Research Program. The authors also thank ISSI for hosting the conference and the Europlanet RI-FP7 project and its related Science Networking (Na2) working groups. Finally, the authors thank guest editor M. Toplis and two anonymous referees for their suggestions and recommendations which helped to improve the article.

\section{References}

T. J. Ahrens, Impact erosion of terrestrial planetary atmospheres. Ann. Rev. Earth Planet. Sci. 21, 525-555(1993)

F. Albarède, J. Blichert-Toft, The split fate of the early Earth, Mars, Venus and Moon. CR Geoscience 339, 917-927 (2007)

U.V. Amerstorfer, N.V. Erkaev, U. Taubenschuss, H.K. Biernat, Influence of a density increase on the evolution of the Kelvin-Helmholtz instability and vortices. Phys. Plasmas 17, 072901, (2010). doi:10.1063/1.3453705

J.C. Andrews-Hanna, M.T. Zuber, W.B. Banerdt, The Borealis basin and the origin of the martian crustal dichotomy. Nature 453, 1212-1215 (2008)

S.K. Atreya, R.P. Mahaffy, A. Wong, A., Methane and related trace species on Mars: Origin, loss, implications for life, and habitability. Planet. Space Sci. 55, 358-369 (2006)

J.L. Bandfield, T.D. Gloch, P.R. Christensen, Spectroscopic identification of carbonate minerals in the martian dust. Science 301, 1084-1086 (2003)

J.L. Bandfield, High-resolution subsurface water-ice distributions on Mars. Nature 447, 64-67 (2007)

S. Barabash, A. Fedorov, R. Lundin, J.-A. Sauvaud, Martian atmospheric erosion rates. Science 315, 501-503 (2007)

R.H. Becker, R.N. Clayton, E.M. Galimov, H. Lammer, B. Marty, R.O. Pepin, R. Weiler, Isotopic signatures in terrestrial planets. Space Sci. Rev. 106, 377-410 (2003)

J.-P. Bibring, Y. Langevin, F. Poulet, A. Gendrin, B. Gondet, M. Berthé, A. Soufflot, P. Drossart, M. Combes, G. Bellucci, V. Moroz, N. Mangold, B. Schmitt, and the OMEGA team. Perennial water ice identified in the south polar cap of Mars. Nature 428, 627-630 (2004)

J.-P. Bibring, Y. Langevin, A. Gendrin, B. Gondet, F. Poulet, M. Berthé, A. Soufflot, R. Arvidson, N. Mangold, J. Mustard, P. Drossart, and the OMEGA team Mars surface diversity as revealed by the OMEGA/Mars Express observations. Science 307, 1576-1581 (2005)

J.-P. Bibring, Y. Langevin, J.F. Mustard, F. Poulet, R. Arvidson, A. Gendrin, B. Gondet, N. Mangold, P. Pinet, F. Forget, and the OMEGA team, Global mineralogical and aqueous Mars history derived from OMEGA/Mars Express data. Science 312, 400-404 (2006)

L.E. Borg, D.S. Draper, A petrogenetic model for the origin and compositional variation of the Martian basaltic meteorites. Meteorit. Planet. Sci. 38, 1713-1731 (2003)

W.F. Bottke, H.F. Levison, D. Nesvorny, L. Dones, Can planetesimals left over from terrestrial planet formation produce the lunar Late Heavy Bombardment? Icarus 190, 203-223 (2007)

W.V. Boynton, W.C. Feldman, S.W. Squyres, T.H. Prettyman, J. Brückner, L.G. Evans, R.C. Reedy, R. Starr, J.R. Arnold, D.M. Drake, P.A.J. Englert, A.E. Metzger, I. Mitrofanov, J.I. Trombka, C. d'Uston, H. Wänke, O. Gasnault, D.K. Hamara, D.M. Janes, R.L. Marcialis, S. Maurice, I. Mikheeva, G.J. Taylor, R. Tokar, C. Shinohara,Distribution of Hydrogen in the Near Surface of Mars: Evidence for Subsurface Ice Deposits. Science 297, 81-85 (2002)

W.V. Boynton, W.C. Feldman, I.G. Mitrofanov, L.G. Evans, R.C. Reedy, S. W. Squyres, R. Starr, J.I. Trombka, C d'Uston, J.R. Arnold, P.A.J. Englert, A.E. Metzger, H. Wänke, J. Brückner, D.M. Drake, C. Shinohara, C. Fellows, D.K. Hamara, K. Harshman, K. Kerry, C. Turner, M. Ward, H. Barthe, K.R. Fuller, S.A. Storms, G.W. Thornton, J.L. Longmire, 
M.L. Litvak, A.K. Ton'chev, The Mars Odyssey gamma-ray spectrometer instrument suite. Space Sci. Rev. 110, 37-83 (2004)

L.H. Brace, R.F. Theis, W.R. Hoegy, Plasma clouds above the ionopause of Venus and their implications. Planet. Space Sci. 30, 29-37 (1982)

R. Brasser, The formation of Mars: Building blocks and accretion time scale. Space Sci. Rev., in press doi:10.1007/s11214-012-9904-2 (2012)

D. Breuer, T. Spohn, Viscosity of the Martian mantle and its initial temperature: Constraints from crust formation history and the evolution of the magnetic field. Planet. Space Sci. 54, 153-169 (2006)

J.C. Bridges, D.C. Catling, J.M. Saxton, T.D. Swindle, I.C. Lyon, M.M. Grady, Alteration assemblages in martian meteorites: Implications for near-surface processes. Space Sci. Rev. 96, 365-392 (2001)

A.G.W.Cameron, Origin of the atmospheres of the terrestrial planets. Icarus 56, 195-201 (1983)

R.M. Canup, E. Asphaug, Origin of the Moon in a giant impact near the end of the Earth's formation. Nature 412, 708-712 (2001)

M.H. Carr, Mars - A water-rich planet? Icarus 68, 187-216 (1986)

M.H. Carr, Retention of an atmosphere on early Mars. J. Geophys. Res. 1042, 21897-21910 (1999)

M.H. Carr, J.W. Head, Oceans on Mars: An assessment of the observational evidence and possible fate, J. Geophys. Res. 108, doi:10.1029/2002JE001963 (2003)

D.C. Catling, Mars: Ancient fingerprints in the clay. Nature 448, 31-32 (2007)

C.R. Chapman, B.A. Cohen, D.H. Grinspoon, What are the real constraints on the existence and magnitude of the Late Heavy Bombardment? Icarus 189, 233-245 (2007)

E. Chassefière, Hydrodynamic escape of oxygen from primitive atmospheres: applications to the cases of Venus and Mars. Icarus 124 537-552 (1996)

E. Chassefière, Metastable methane clathrate particles as a source of methane to the Martian atmosphere. Icarus 204 137-144 (2009)

E. Chassefière, F. Leblanc, Mars atmospheric escape and evolution, interaction with the solar wind. Planet. Space Sci. 52, 1039-1058 (2004)

E. Chassefière, F. Leblanc, B. Langlais, The combined effects of escape and magnetic field histories at Mars. Planet. Space Sci. 55, 343-357 (2007)

E. Chassefière, F. Leblanc, Constraining methane release due to serpentinization by the observed D/H ratio on Mars. Earth Planet. Sci. Lett. 310 262-271 (2011a)

E. Chassefière, F. Leblanc, Explaining the redox imbalance between the $\mathrm{H}$ and $\mathrm{O}$ escape fluxes at Mars by the oxidation of methane, Planet. Space Sci. 59 218-226 (2011b)

E. Chassefière, F. Leblanc, Constraining methane release due to serpentinization by the observed D/H ratio on Mars. Earth Planet. Sci. Lett. 310, 262-271 (2011c)

Chastain, B. K., Chevrier, V., 2007. Methane clathrate hydrates as a potential source for Martian atmospheric methane, Planet. Space Sci. 55, 1246-1256 (2007)

J. Y. Chaufray, R. Modolo, F. Leblanc, G. Chanteur, R.E. Johnson, J.G. Luhmann, Mars solar wind interaction: Formation of the Martian corona and atmospheric loss to space. J. Geophys. Res. 112, E9, CiteID E09009, (2007)

V. Chevrier, F. Poulet, J.-P. Bibring, Early geochemical environment of Mars as determined from thermodynamics of phyllosilicates. Nature 448, 60-63 (2007)

P. Christensen, Water at the Poles and in Permafrost Regions of Mars. Elements 2, 151-155 (2006)

C.F. Chyba, T.C. Owen, W.-H. IP, Impact delivery of volatiles and organic to molecules to Earth, in Hazards due to comets and asteroids, ed. by T. Gehrels, M.S. Matthews, A. Schumann (Space Science Series, University of Arizona Press, Tucson, 1994) pp. 9-58

M.W. Claire, J. Sheets, M. Cohen, I. Ribas, V. S. Meadows, D. C. Catling, The evolution of solar flux from $0.1 \mathrm{~nm}$ to $160 \mu \mathrm{m}$ : Quantitative estimates for planetary studies. ApJ 757, 95 12pp (2012)

S.M. Clifford, J. Lasue, E. Heggy, J. Boisson, P. McGovern, M.D. Max, Depth of the Martian cryosphere: Revised estimates and implications for the existence and detection of subpermafrost groundwater. J. Geophys. Res. 115, E07001, doi:10.1029/2009JE003462 (2010)

R.A. Craddock, R. Greeley, Minimum estimates of the amount and timing of gases released into the martian atmosphere from volcanic eruptions. Icarus 204, 512-526 (2009)

N. Dauphas, The dual origin of the terrestrial atmosphere. Icarus 165, 326-339 (2003) 
N. Dauphas, A. Pourmand, Hf-W-Th evidence for rapid growth of Mars and its status as a planetary embryo, Nature 473, 489-493 (2011)

V. Dehant, H. Lammer, Y.N. Kulikov, J.-M. Griemeier, D. Breuer, O. Verhoeven, O. Karatekin, T. van Hoolst, O. Korablev, P. Lognonne, Planetary magnetic dynamo effect on atmospheric protection of early Earth and Mars. Space Sci. Rev. 129, 279-300 (2007)

J.M. Dohm, R.C. Anderson, N.G. Barlow, H. Miyamoto, A.G. Davies, G.J. Taylor, V.R. Baker, W.V. Boynton, J. Keller, K. Kerry, D. Janes, A.G. Fairén, D. Schulze-Makuch, M. Glamoclija, L. Marinangeli, G.G. Ori, R.G. Strom, J.-P. Williams, J.C. Ferris, J.A.P. Rodríguez, M.A. de Pablon, S. Karunatillake, Recent geological and hydrological activity on Mars: The Tharsis/Elysium corridor. Planet. Space Sci. 56, 985-1013 (2008)

T.M. Donahue, Evolution of water reservoirs on Mars from D/H ratios in the atmosphere and crust. Nature 374, 432-434 (1995)

T.M. Donahue, Pre-Global Surveyor evidence for Martian ground water. PNAS 98, 827-830 (2001)

T.M. Donahue, Accretion, loss, and fractionation of martian water. Icarus 167, 225-227 (2004)

J. D. Dorren, M. Güdel, E.F. Guinan, X-ray emission from the Sun in its youth and old age. Astrophys. J. 448, 431-436 (1995)

B. L. Ehlmann, J.F. Mustard, S.L. Murchie, F. Poulet, J.L. Bishop, A.J. Brown, W.M. Calvin, R.N. Clark, D.J. des Marais, R.E. Milliken, L.H. Roach, T.L. Roush, G.A. Swayze J.J. Wray, Orbital identification of Carbonate-bearing rocks on Mars. Science 322, 1828-1832 (2008)

B. L. Ehlmann, J.F. Mustard, G.A. Swayze, R.N. Clark, J.L. Bishop, F. Poulet, D.J. des Marais, L.H. Roach, R.E. Milliken, J.J. Wray, O. Barnouin-Jha, S.L. Murchie, Identification of hydrated silicate minerals on Mars using MRO-CRISM: Geologic context near Nili Fossae and implications for aqueous alteration. J. Geophys. Res. E00D08 (2009). doi:10.1029/2009JE003339

B. L. Ehlmann, et al. Geochemical consequences of widespread clay formation in Mars' Ancient Crust, Space Sci. Rev., this issue, (2012)

Elkins-Tanton LT (2008) Linked magma ocean solidification and atmospheric growth for Earth and Mars. Earth and Planet Sci Lett 271:181-191 (2008)

O. Eugster, H. Busemann, S. Lorenzetti, D. Terrebilini, Ejection ages from krypton-81-krypton83 dating and pre- atmospheric sizes of martian meteorites. Meteorite Planet. Sci. 37, 1345-1360 (2002)

A.G.Fairén, A cold and wet Mars. Icarus 208, 165-175 (2010)

W.C. Feldman, W.V. Boynton, R.L. Tokar, T.H. Prettyman, O. Gasnault, S.W. Squyres, R.C. Elphic, D.J. Lawrence, S.L. Lawson, S. Maurice, G.W. McKinney, K.R. Moore, R.C. Reedy, Global distribution of neutrons from Mars: Results from Mars Odyssey. Science 297, 75-78 (2002)

J. Filiberto, A.H. Treiman, Martian magmas contained abundant chlorine, but little water. Geology 37, 1087-1090 (2009)

S. Fonti, G.A. Marzo, Mapping the methane on Mars. Astron. Astrophys. 512, A51 (2010)

V. Formisano, S. Atreya, T. Encrenaz, N. Ignatiev, M. Giuranna, Detection of methane in the atmosphere of Mars. Science 306, 1758-1761 (2004)

J.L. Fox, $\mathrm{CO}_{2}^{+}$dissociative recombination: a source of thermal and nonthermal C on Mars. J. Geophys. Res. 109, CiteID A08306 (2004)

J.L. Fox, A.B. Hać, Photochemical escape of oxygen from Mars: A comparison of the exobase approximation to a Monte Carlo method. Icarus 204, 527-544 (2009)

A.A. Fraeman, J. Korenaga, The influence of mantle melting on the evolution of Mars. Icarus 210, 43-57 (2010)

H. V. Frey, Impact constraints on, and a chronology for, major events in early Mars history. J. Geophys. Res. 111, E08S91 (2006)

E. Gaidos, G. Marion, Geological and geochemical legacy of a cold early Mars. J. Geophys. Res. 108, E6, CiteID 5055, pp. 9-1 (2003)

A. Geminale, V. Formisano, M. Giuranna, Methane in Martian atmosphere: Average spatial, diurnal, and seasonal behaviour. Planet. Space Sci. 56, 1194-1203 (2008)

A. Geminale, V. Formisano, G. Sindoni, Mapping methane in Martian atmosphere with PFSMEX data. Planet. Space Sci. 59, 137-148 (2011)

A. Gendrin, N. Mangold, J.-P. Bibring, Y. Langevin, B. Gondet, F. Poulet, G. Bonello, C. Quantin, J. Mustard, R. Arvidson, S LeMouélic, Sulfates in martian layered terrains: The OMEGA/Mars Express view. Science 307, 1587-1591 (2005) 
T.M. Gerlach, E.J. Graeber, Volatile budget of Kilauea volcano. Nature 313, 273-277 (1985)

S.R. Gíslason, et al. Effects of volcanic eruptions on the $\mathrm{CO}_{2}$ content of the atmosphere and the oceans: the 1996 eruption and flood within the Vatnajökull Glacier, Iceland. Chem. Geol. 190, 181-205 (2002)

R. Gomes, H.F. Levison, K. Tsiganis, A. Morbidelli, Origin of the cataclysmic Late Heavy Bombardment period of the terrestrial planets. Nature 435, 466-469 (2005)

J.L. Gooding, S.J. Wentworth, M.E. Zolensky, Calcium-Carbonate and Sulfate of possible extraterrestrial origin in the Eeta-79001 meteorite. Geochim. Cosmochimi. Acta 52, 909915 (1988)

R.Greeley, B.B. Schneid, Magma generation on Mars -amounts, rates and comparisons with Earth, Moon, and Venus. Science 254, 996-998 (1991)

L.P. Greenland, Composition of gases from the 1984 eruption of Mauna Loa volcano, in Volcanism in Hawaii, vol. 1, U.S. Geol. Surv. Prof. Pap. 1350. U.S. Gov. Printing Office, Washington, 781-790 (1987a)

L.P. Greenland, Hawaiian eruptive gases, in Volcanism in Hawaii, vol. 1, U.S. Geol. Surv. Prof. Pap. 1350. U.S. Gov. Printing Office, Washington, 781-790 (1987b)

L.L. Griffith, E.L. Shock, A geochemical model for the formation of hydrothermal carbonates on Mars. Nature 377, 406-408 (1995)

L.L. Griffith, E.L. Shock, Hydrothermal hydration of Martian crust: Illustration via geochemical model calculations. J. Geophys. Res. 102, 9135-9143 (1997)

H. Gröller, V.I. Shematovich, H.I.M. Lichtenegger, H. Lammer, M. Pfleger, Yu.N. Kulikov, W. Macher, U.V. Amerstorfer, H.K. Biernat, Venus' atomic hot oxygen environment. J. Geophys. Res. 115, CiteID, E12017 (2010)

H. Gröller, H. Lammer, H.I.M. Lichtenegger, M. Pfleger, O. Dutuit, V.I. Shematovich, Yu.N. Kulikov, H.K. Biernat, Hot oxygen atoms in the Venus nightside exosphere. Geophys. Res. Lett. 39, Issue 3 (2012)

M. Grott, A. Morschhauser D. Breuer, E. Hauber, Volcanic outgassing of $\mathrm{CO}_{2}$ and $\mathrm{H}_{2} \mathrm{O}$ on Mars. Earth Planet. Sci. Lett. 308, 391-400 (2011)

M. Grott, D. Baratoux, E. Hauber, D. Breuer, S.-I. Karato, M. Knapmeyer, J. Mustard, S. Ruff, F. Sohl, V. Sautter, M. Toplis, O. Gasnault, S.M. McLennan, Long-term evolution of the crust-mantle system. Space Sci. Rev., this issue (2012)

M. Güdel, E.F. Guinan, R. Mewe, J.S. Kaastra, S.L. Skinner, A determination of the coronal emission measure distribution in the young solar analog EK Draconis from ASCA/EUVE spectra. Astrophys. J. 479, 416-426, (1997)

A.N. Halliday, The origin of the earliest history of the Earth. Treatise Geochem. 1, 509-557 (2003)

K. Hamano, Y. Abe, Impact erosion of atmospheres: Substantial atmospheric loss by horizontal atmospheric excavation, in Proc. Role of volatiles and atmospheres on martian impact craters workshop 2005, pp. 46-47

K. Hamano, Y. Abe, Pressure dependence of atmospheric loss by impact-induced vapor expansion, in The 37th Lunar and Planetary Science Conference, abs. 1562 (2006)

P. Hartogh, D.C. Lis, D. Bockelée-Morvan, M. de Val-Borro, N. Biver, M. Küppers, M. Emprechtinger, E.A. Bergin, J. Crovisier, M. Rengel, R. Moreno, Raphael, S. Szutowicz, G.A. Blake, Geoffrey, Ocean-like water in the Jupiter-family comet 103P/Hartley 2. Nature 478, 218-220 (2011)

E. Hauber, P. Broẑ̀, F. Jagert, P. Jodlowski, T. Platz, Very recent and wide-spread basaltic volcanism on Mars, Geophys. Res. Lett. 38, L10201 (2011)

S.A. Hauck II, R.J. Phillips, Thermal and crustal evolution of Mars. J. Geophys. Res., 107, 505219 pp (2002)

C. Hayashi, K. Nakazawa, H. Mizuno, Earth's melting due to the blanketing effect of the primordial dense atmosphere. Earth Planet. Sci. Lett. 43 22-28 (1979)

J.W. Head, S. Pratt, Extensive Hesperian-aged south polar ice sheet on Mars: Evidence for massive melting and retreat, and lateral flow and ponding of meltwater. J. Geophys. Res. 106, 12275-12300 (2001)

J.W. Head, R. Greeley, M.P. Golombek, W.K. Hartmann, E. Hauber, R. Jaumann, P. Masson, G. Neukum, L.E. Nyquist, M.H. Carr, Geological Processes and Evolution. Space Sci. Rev. 96, 263-292 (2001)

J.W. Head, D.R. Marchant, Inventory of Ice-related deposits on Mars: Evidence for burial and long-term sequestration of ice in non-polar regions and implications for the water budget and climate evolution, in The $40^{\text {th }}$ Lunar and Planetary Science Conference, abs. 1356 
(2009)

C.D.K. Herd, L.E. Borg, J.H. Jones, J.J. Papike, Oxygen fugacity and geochemical variations in the martian basalts: implications for martian basalt petrogenesis and the oxidation state of the upper mantle of Mars. Geochim. Cosmochim. Acta 66, 2025-2036 (2002)

M.M. Hirschmann, A.C. Withers, Ventilation of $\mathrm{CO}_{2}$ from a reduced mantle and consequences for the early Martian greenhouse. Earth Planet. Sci. Lett. 270, 147-155 (2008)

J.R. Holloway, V. Pan, G. Gudmundsson, High-pressure fluid-absent melting experiments in the presence of graphite: oxygen fugacity, ferric/ferrous ratio and dissolved $\mathrm{CO}_{2}$. Eur. J. Mineral. 4, 105-114 (1992)

J.R. Holloway, Graphite melt equilibria during mantle melting: constraints on $\mathrm{CO}_{2}$ in $\mathrm{MORB}$ magmas and the carbon content of the mantle. Chem. Geol. 147, 89-97 (1998)

J. Horner, O. Mousis, J.-M. Petit, B.-W. Jones, Differences between the impact regimes of the terrestrial planets: Implications for primordial D:H ratios. Planet. Space Sci. 57, 1338-1345 (2009)

D.M. Hunten, R.O. Pepin, J.C.G. Walker, Mass fractionation in hydrodynamic escape. Icarus 69, 532-549 (1987)

W.-H. Ip, On a hot oxygen corona of Mars. Icarus, 76, 135-145 (1988)

N. Iro, D. Gautier, F. Hersant, D. Bockelée-Morvan, J.-I. Lunine, An interpretation of the nitrogen deficiency in comets. Icarus 161, 511-532 (2003)

B.A. Ivanov, Mars/Moon cratering rate ratio estimates. Space Sci. Rev. 96, 87-104 (2004)

B.A. Ivanov, Cratering rate comparisons between terrestrial planets, in Proc. Workshop on surface ages and histories: Issues in planetary chronology, 2006, pp. 26-27

B.A. Ivanov, V.V. Shuvalov, N.A. Artemieva, Meteoritic bombardment in the Noachian time: Influence on geological and atmospheric evolution, in Proc. $35^{\text {th }}$ Microsymposium of the Brown University and the Vernadsky Institute, 2002

B.M. Jakosky, B. M. Mars volatile evolution: Evidence from stable isotopes. Icarus 94, 14-31 (1991)

B.M. Jakosky, R.O. Pepin, R.E. Johnson, J.L. Fox, Mars atmospheric loss and isotopic fractionation by solar-wind-induced sputtering and photochemical escape. Icarus 111, 271-288 (1994)

M.C. Johnson, M.J. Rutherford, P. C. Hess, Chassigny petrogenesis: Melt compositions, intensive parameters and water contents of Martian magmas. Geochim. Cosmochim. Acta 55, 349-366 (1991)

J.F. Kasting, J.B. Pollack, Loss of water from Venus I. Hydrodynamic escape of hydrogen. Icarus 53, 479-508 (1983)

J.F. Kasting, $\mathrm{CO}_{2}$ condensation and the climate of early Mars. Icarus 94, 1-13 (1991)

J.F. Kasting, The early Mars climate question heats up. Science 278, p. 1245 (1997)

R.F. Katz, M. Spielman, C.H. Langmuir, A new parameterization of hydrous mantle melting. Geochem. Geophys. Geosyst. 4, 1073, (2003)

H.H. Kieffer, S.C. Chase Jr., T.Z. Martin, E.D. Miner, F. Don Palluconi, Martian north pole summer temperatures: Dirty water ice. Science 194, 1341-1344 (1976)

J. Kim, A.F. Nagy, J.L. Fox, T.E. Cravens, Solar cycle variability of hot oxygen atoms at Mars. J. Geophys. Res., 103, 29339-29342 (1998)

T.T. Koskinen, M.J. Harris, R.V., Yelle, P., Lavvas, The escape of heavy atoms from the ionosphere of HD 209458b. I. A photochemical-dynamical model of the thermosphere. Icarus, submitted. (2012)

V.A. Krasnopolsky, G.L. bjoraker, M.J. Mumma, D.F. Jennings, High resolution spectroscopy of Mars at 3.7 and $8 \mu \mathrm{m}$. J. Geopys. Res. 102, 6525-6534 (1997)

V.A. Krasnopolsky, M.J. Mumma, G.R. Gladstone, Detection of atomic Deuterium in the upper atmosphere of Mars. Science 280, 1576-1580 (1998)

V.A. Krasnopolsky, P.D. Feldman, Detection of molecular hydrogen in the atmosphere of Mars. Science 294, 1914-1917 (2001)

V.A. Krasnopolsky, Some problems related to the origin of methane on Mars. Icarus 180, 359-367 (2006)

V.A. Krasnopolsky, J.-P. Maillard, T.C. Owen, Detection of methane in the Martian atmosphere: evidence for life?, Icarus 172, 537-547 (2004)

M.A. Krestyanikova, V. I. Shematovich, Stochastic models of hot planetary and satellite coronas: A hot oxygen corona of Mars. Solar System Res. 40, 384-392 (2006)

H. Lammer, W. Stumptner, S.J. Bauer, Upper limits for the Martian exospheric number density during the planet B/Nozomi mission. Planet. Space Sci. 48, 1473-1478 (2000) 
H. Lammer, C. kolb, T. Penz, U.V. Amerstorfer, H. K. Biernat, B. Bodiselitsch, Estimation of the past and present martian water-ice reservoirs by isotopic constraints on exchnage between the atmosphere and the surface. Int. J. Astrobiol. 2(3), 195-202 (2003)

H. Lammer, H.I.M. Lichtenegger, H.K. Biernat, N.V. Erkaev, I. Arshukova, C. Kolb, H. Gunell, A. Lukyanov, M. Holmstrom, S. Barabash, T.L. Zhang, W. Baumjohann, Loss of hydrogen and oxygen from the upper atmosphere of Venus. Planet. Space Sci. 54, 1445-1456 (2006)

H. Lammer, J.F. Kasting, E. Chassefière, R.E. Johnson, Y.N. Kulikov, F. Tian, Atmospheric escape and evolution of terrestrial planets and satellites. Space Sci. Rev., 139, 399-436 (2008)

H. Lammer, P. Odert, M. Leitzinger, M.L. Khodachenko, M. Panchenko, Yu.N. Kulikov, T.L. Zhang, H.I.M. Lichtenegger, N.V. Erkaev, G. Wuchterl, G. Micela, T. Penz, H.K. Biernat, J. Weingrill, M. Steller, H. Ottacher, J. Hasiba, A. Hanslmeier, Determining the mass loss limit for close-in exoplanets: What can we learn from transit observations? Astron Astrophys 506, 399-410 (2009)

H. Lammer, K. G. Kislyakova, P. Odert, M. Leitzinger, R. Schwarz, E. Pilat-Lohinger, Yu. N. Kulikov, M. L. Khodachenko, M. Güdel, A. Hanslmeier, Pathways to Earth-like atmospheres: extreme ultraviolet (EUV)-powered escape of hydrogen-rich protoatmospheres. Orig. Life Evol. Biosph. 41, 503-522 (2012)

Y. Langevin, F. Poulet, F., Bibring, J.-P., B. Gondet, Sulfates in the north polar region of Mars detected by OMEGA/Mars Express. Science 307, 1584-1587 (2005)

J. Lasue, N. Mangold, E. Hauber, S. Clifford, W. Feldman, O. Gasnault, C. Grima, S. Maurice, O. Mousis, Quantifying the Martian hydrosphere: current evidence, time evolution and implications for the habitability of the planet. Space Sci. Rev., submitted (2012)

F. Leblanc, R. E. Johnson, Role of molecular species in pick up ion sputtering of the Martian atmosphere. J. Geophys. Res. 107, 1-6 (2002)

F. Lefèvre, F. Forget, Observed variations of methane on Mars unexplained by known atmospheric chemistry and physics. Nature 460, 720-723 (2002)

L.A. Leshin, S. Epstein, E.M. Stolper, Hydrogen isotope geochemistry of SNC meteorites. Geochim. Cosmochim. Acta 60, 2635-2650 (1996)

04 B. Levrard, F. Forget, F. Montmessin, J. Laskar, Ice-rich deposits formed at high latitude on Mars by sublimation of unstable equatorial ice during low obliquity. Nature 431, 1072$1075(2004)$

L.-G. Liu, The inception of the oceans and $\mathrm{CO}_{2}$-atmosphere in the early history of the Earth. Earth Planet. Sci. Lett. 227, 179-184 (2004)

J.G. Luhmann, J. U. Kozyra, Dayside pickup oxygen ion precipitation at Venus and Mars: spatial distributions, energy deposition and consequences. J. Geophys. Res. 96, 5457-5467 (1991)

J.G. Luhmann, R.E. Johnson, M.H.G. Zhang, Evolutionary impact of sputtering of the Martian atmosphere by $\mathrm{O}(+)$ pickup ions. Geophys. Res. Lett. 19, 2151-2154 (1992)

J.I. Lunine, J. Chambers, A. Morbidelli, L.A. Leshin, The origin of water on Mars. Icarus 165, 1-8 (2003)

J.I. Lunine, D.P. O`Brien, S.N. Raymond, A. Morbidelli, T. Qinn, A.L. Graps, Dynamical models of terrestrial planet formation. Adv. Sci. Lett. 4, 325-338 (2011)

R. Lundin, H. Lammer, I. Ribas, Planetary magnetic fields and solar forcing: Implications for atmospheric evolution. Space Sci. Rev. 129, 245-278 (2007)

R. Lundin, Ion acceleration and outflow from Mars and Venus: An overview. Space Sci. Rev., in press, DOI 10.1007/s11214-011-9811-y

J. Lyons, C. Manning, F. Nimmo, Formation of methane on Mars by fluid-rock interaction in the crust. Geophys. Res Lett. 32, 13, L13201.1-L13201.4 (2005)

Y.-J. Ma, A.F. Nagy, Ion escape fluxes from Mars. Geophys. Res. Lett. 34, L08201, (2007)

H.Y. McSween, P.P. Harvey, Outgassed water on Mars: Constraints from melt inclusions in SNC meteorites. Science 259, 1890-1892 (1993)

H.Y. McSween, K. Keil, Mixing relationships in the Martian regolith and the composition of globally homogeneous dust. Geochi. Cosmochim. Acta 64, 2155-2166 (2000)

H.Y. McSween, T.L. Grove, R.C. Lentz, J.C. Dann, A.H. Holzheid, L.R. Riciputi, J.G. Ryan, Geochemical evidence for magmatic water within Mars from pyroxenes in the Shergotty meteorite. Nature 409, 487-490 (2001)

N. Mangold, V. Ansan, Detailed study of an hydrological system of valleys, a delta and lakes in the Southwest Thaumasia region, Mars. Icarus 180, 75-87 (2006) 
C.V. Manning, C.P. McKay, K.J. Zahnle, Thick and thin models of the evolution of carbon dioxide on Mars. Icarus 180, 38-59 (2006)

C.V. Manning, Y. Ma, D.A. Brain, C.P. McKay, K.J. Zahnle, Parametric analysis of modeled ion escape from Mars. Icarus 212, 131-137, (2010)

B. Marty, A. Meibom, Noble gas signature of the late heavy bombardment in the Earth's atmosphere. Earth Discussions 2, 99-113 (2007)

T. Matsui, Y. Abe, Impact-induced atmospheres and oceans on Earth and Venus. Nature 322, 526-528 (1986)

T. McCollom, W. Back, Thermodynamic constraints on hydrogen generation during serpentinization of ultramafic rocks, Geochim. Cosmochim. Acta 73, 856-875 (2009)

W.B. McKinnon, Impacts giveth and impacts taketh away. Nature 338, 465-466 (1989)

H.J. Melosh, A.M. Vickery, Impact erosion of the primordial atmosphere of Mars. Nature 338, 487-489 (1989)

J.R. Michalski, P.B. Niles, Deep crustal carbonate rocks exposed by meteor impact on Mars. Nature Geosci. 3, 751-755 (2010)

I.G. Mitrofanov, D. Anfimov, A. Kozyrev, M. Litvak, A. Sanin, V.I. Tret'yakov, A. Krylov, V. Shvetsov, W. Boynton, C. Shinohara, D. Hamara, R.S. Saunders, Maps of subsurface hydrogen from the High Energy Neutron Detector, Mars Odyssey. Science 297, 78-81 (2002)

I.G. Mitrofanov, M.L. Litvak, A.S. Kozyrev, A.B. Sanin, V.I. Tret'yakov, V. Yu. Grin'kov, W.V. Boynton, C. Shinohara, C., D. Hamara, R.S. Saunders, Soil water content on Mars as estimated from neutron measurements by the HEND instrument onboard the 2001 Mars Odyssey Spacecraft. Solar Syst. Res. 38, 253-257 (2004)

D.W. Mittlefehldt, ALH84001, a cumulate orthopyroxenite member of the Martian meteorite clan. Meteoritics 29, 214-221 (1994)

H. Mizuno, K. Nakazawa, C. Hayashi, Dissolution of the primordial rare gases into the molten Earth' material. Earth Planet. Sci. Lett. 50, 202-210 (1980)

A. Morbidelli, J. Chambers, J.I. Lunine, J.M. Petit, F. Robert, G.B. Valsecchi, K. Cyr, Source regions and timescales for the delivery of water to Earth. Meteorit. Planet. Sci. 35, 1309$1320(2000)$

R. Modolo, G.M. Chanteur, E. Dubinin, A.P. Matthews, Influence of the solar EUV flux on the Martian plasma environment. Ann. Geophysicae 23, 1-12 (2005)

A. Morschhauser, M. Grott, D. Breuer, Crustal recycling, mantle dehydration, and the thermal evolution of Mars. Icarus 212, 541-558 (2011)

R. V. Morris, S. W. Ruff, R. Gellert, D.W. Ming, R.E. Arvidson, B.C. Clark, D.C. Golden, K.Siebach, G. Klingelhöfer, C. Schröder, I. Fleischer, A.S. Yen, S.W. Squyres, Identification of Carbonate-Rich Outcrops on Mars by the Spirit Rover. Science 329, 421-424 (2010)

O. Mousis, J.I. Lunine, J.-M. Petit, S. Picaud, B. Schmitt, D. Marquer, J. Horner, C. Thomas, Impact regimes and post-formation sequestration processes: Implications for the origin of heavy noble gases in terrestrial planets. Astrophys. J. 714, 1418-1423 (2010)

O. Mousis, J.I. Lunine, E. Chassefière, F. Montmessin, A. Lakhlifi, S. Picaud, J.M. Petit, D. Cordier, Mars cryosphere: A potential reservoir for heavy noble gases? Icarus 218, 80-87 (2012)

D. Möhlmann, Widen the belt of habitability! Orig. Life Evol. Biosph. 42, 93-100 (2012)

U.V. Möstl, N.V. Erkaev, M. Zellinger, H. Lammer, H. Gröller, H.K. Biernat, D. Korovinskiy, The Kelvin-Helmholtz instability at Venus: What is the unstable boundary? Icarus 216, 476-484 (2011)

M.J. Mumma, G.L. Villanueva, R.E. Novak, T. Hewagama, B.P. Bonev, M.A. DiSanti, A.M. Mandell, D.M. Smith, Strong Release of Methane on Mars in Northern Summer 2003. Science 323, 1041-1045 (2009)

J.F.Mustard, S.L. Murchie, S.M. Pelkey, B.L. Ehlmann, R.E. Milliken, J.A. Grant, J.-P. Bibring, F. Poulet, J. Bishop, E. Noe Dobrea, L. Roach, F. Seelos, R.E. Arvidson, S.R. Green, H. Hash, D. Humm, E. Malaret, J.A. McGovern, K. Seelos, T. Clancy, R. Clark, D.D. Marais, N. Izenberg, A. Knudson, Y. Langevin, T. Martin, P. McGuire, R. Morris, M. Robinson, T. Roush, M. Smith, G. Swayze, H. Taylor, T. Titus, M. Wolff, Hydrated silicate minerals on Mars observed by the Mars Reconnaissance Orbiter CRISM instrument. Nature 454, 305-309 (2008)

S.L. Murchie, J.F. Mustard, B.L. Ehlmann, R.E. Milliken, J.L. Bishop, N.K. McKeown, E.Z. Noe Dobrea, F.P. Seelos, D.L. Buczkowski, S.M. Wiseman, R.E. Arvidson, J.J. Wray, G. Swayze, R.N. Clark, D.J. Des Marais, A.S. McEwen, and J.-P. Bibring, A synthesis of Mar- 
tian aqueous mineralogy after 1 Mars year of observations from the Mars Reconnaissance Orbiter. J. Geophys. Res. 114, E00D06, 30 pp. (2009)

A.F. Nagy, J. Kim, T.E. Cravens, Hot hydrogen and oxygen atoms in the upper atmospheres of Venus and Mars. Ann. Geophysicae 8, 251-256, (1990)

G. Neukum, D.U. Wise. Mars: A standard crater curve and possible new time scale. Science 194, 1381-1387 (1976)

G. Neukum, B.A. Ivanov, W.K. Hartmann, Cratering records in the inner Solar System in relation to the Lunar reference system. Space Sci. Rev. 96, 55-86 (2001)

G. Newkirk Jr., Solar variability on time scales of $10^{5}$ years to $10^{9.6}$ years. Geochi. Cosmochi. Acta Suppl. 13, 293-301, (1980)

W.I. Newman, E.M.D. Symbalisty, T.J. Ahrens, E.M. Jones, Impact Erosion of Planetary Atmospheres: Some Surprising Results. Icarus 138, 224-240 (1999)

P.B. Niles, W.V. Boynton, J.H. Hoffman, D.W. Ming, D. Hamara, Stable isotope measurements of martian atmospheric $\mathrm{CO}_{2}$ at the Phoenix landing site. Science 329, 1334-1337 (2010)

P.B. Niles, D.C. Catling, G. Berger, E. Chassefière, B.L. Ehlmann, J.R. Michalski, R. Morris, S.W. Ruff, B. Sutter, Geochemistry of Carbonates on Mars: Implications for Climate History and Nature of Aqueous Environments. Space Science Rev., submitted (2012)

M.D. Norman, The composition and thickness of the crust of Mars estimated from REE and Nd isotopic compositions of Martian meteorites. Meteorit. Planet. Sci. 34, 439-449 (1999)

L.E. Nyquist, D.D. Bogard, C.-Y. Shih, A. Greshake, D. Stöffler, O. Eugster, Ages and geologic histories of martian meteorites. Space Sci. Rev. 96, 105-164 (2001)

D. Olsson-Steel, Collisions in the solar system. IV: Cometary impacts upon the planets. Month. Not. Royal Astron. Soc. 227, 501-524 (1987)

T. Owen, A. Bar-Nun, Comets, impacts and atmospheres. Icarus 116, 215-226 (1995)

T. Owen, A. Bar-Nun, I. Kleinfeld, Possible cometary origin of heavy noble gases in the atmospheres of Venus, Earth, and Mars. Nature 358, 43-46 (1992)

J.E. Owen, A.P. Jackson, Planetary evaporation by UV \& X-ray radiation: basic hydrodynamics, accepted for publication in Mont. Notes Royal Astron. Soc. (2012)

C. Oze, M. Sharma, Have olivine, will gas : Serpentinization and the abiogenic production of methane on Mars. Geophys. Res. Lett. 32, L10203 (2005)

C. O'Neill, A. Lenardic, A.M. Jellinek, W.S. Kiefer, Melt propagation and volcanism in mantle convection simulations, with applications for Martian volcanic and atmospheric evolution. J. Geophys. Res. 112, E07003 (2007)

D.P. Page, Recent low-latitude freeze thaw on Mars. Icarus 189, 83-117 (2007)

T. Penz, N.V. Erkaev, H.K. Biernat, H. Lammer, U.V. Amerstorfer, H. Gunell, E. Kallio, S. Barabash, S. Orsini, A. Milillo, W. Baumjohann, Ion loss on Mars caused by the KelvinHelmholtz instability. Planet. Space Sci. 52, 1157-1167 (2004)

R.O. Pepin, On the origin and early evolution of terrestrial planet atmospheres and meteoritic volatiles. Icarus 92, 2-79 (1991)

R.O. Pepin, Evolution of Earth's Noble Gases: Consequences of Assuming Hydrodynamic Loss Driven by Giant Impact. Icarus 126, 148-156 (1997)

R.O. Pepin, Atmospheres on the terrestrial planets: Clues to origin and evolution Earth Planet. Sci. Lett. 252, 1-14 (2006)

E. Pierazzo, G. Collins, A brief introduction to hydrocode modeling of impact cratering, in Submarine craters and ejecta-crater correlation, ed. P. Claeys, D. Henning (Springer, New York, 2003), pp. 323-340

L.B.S. Pham, Ö Karatekin, V. Dehant, Effect of meteorite impacts on the atmospheric evolution of Mars. Astrobiology 9, 45-54 (2009)

L.B.S. Pham, Ö. Karatekin, V. Dehant, Effect of an meteorites and asteroids bombardments on the atmospheric evolution of Mars. EPSC Proceedings 5, EPSC2010-127, 2 p. (2010)

L.B.S. Pham, Ö. Karatekin, V. Dehant, Effects of impacts on the atmospheric evolution: comparison between Mars, Earth and Venus. Planet. Space Sci. 59, 1087-1092 (2011)

R.J. Phillips, M.T. Zuber, S.C. Solomon, M.P. Golombek, B.M. Jakosky, W.B. Banerdt, D.E. Smith, R.M.E. Williams, B.M. Hynek, O. Aharonson S.A. Hauck II, Ancient geodynamics and global-scale hydrology on Mars. Science 291, 2587-2591 (2001)

R.J. Phillips, B.J. Davis, S. Byrne, B.A. Campbell, L.M. Carter, R.M. Haberle, J.W. Holt, M.A. Kahre, D.C. Nunes, J.J. Plaut, N.E. Putzig, I.B. Smith, S.E. Smrekar, K.L. Tanaka, T.N. Titus, SHARAD finds voluminous $\mathrm{CO}_{2}$ ice sequestered in the martian South Polar layered deposits. AGU, Fall Meeting, abs. P34A-01.2010 (2010) 
R.J. Phillips, B.J. Davis, K.L. Tanaka, S.M. Byrne, T. Michael, N.E. Putzig, R.M. Haberle, M.A. Kahre, A. Melinda, B.A. Campbell, L.M. Carter, I.B. Smith, J.W. Holt, S.E. Smrekar, D.C. Nunes, J.J. Plaut, Egan, A. F., T.N. Titus, R. Seu, Massive $\mathrm{CO}_{2}$ ice deposits sequestered in the South Polar layered deposits of Mars. Science 332, 838-841 (2011)

R. Pierrehumbert, E. Gaidos, Hydrogen greenhouse planets beyond the habitable zone. ApJ 734, L13 (2011)

J.B. Pollack, J.F. Kasting, S.M. Richardson, K. Poliakoff, The case for a wet, warm climate on early Mars. Icarus 71, 203-224 (1987)

F. Poulet, J.-P. Bibring, J.F. Mustard, A. Gendrin, N. Mangold, Y. Langevin, R.E. Arvidson, B. Gondet, C. Gomez and the Omega Team, Phyllosilicates on Mars and implications for early martian climate. Nature 438, 623-627 (2005)

Y. Quesnel, C. Sotin, B. Langlais, S. Costin, M. Mandea, M. Gottschalk, J. Dyment, Serpentinization of the martian crust during Noachian. Earth Planet. Sci. Lett. 277, 184-193 (2009)

R.R. Rafikov, Atmospheres of protoplanetary cores: critical mass for nucleated instability. ApJ 648, 666-682 (2006)

I. Ribas, E.F. Guinan, M. Güdel, M. Audard, Evolution of the solar activity over time and effects on planetary atmospheres. I. High-energy irradiances (1-1700^). Astrophys. J. 622, 680-694 (2005)

C. Sagan, O.B. Toon, P.J. Gierasch, Climatic change on Mars. Science 181, 1045-1049 (1973)

T.L. Segura, O.B. Toon, A. Colaprete, K. Zahnle, Environmental effects of large impacts on Mars. Science 298, 1977-1980 (2002)

R. Shaheen, A. Abramian, J. Horn, G. Dominguez, R. Sullivan, M.H. Thiemens, Detection of oxygen isotopic anomaly in terrestrial atmospheric carbonates and its implications to Mars. PNAS 107, 20213-20218 (2010)

C.K. Shearer, G. McKay, J.J. Papike, J.M. Karner, Valence state partitioning of vanadium between olivine liquid: estimates of the oxygen fugacity of Y980459 and application to other olivine phyric Martian basalts. Am. Mineral. 91, 1657-1663 (2006)

B.D. Stanley, M.M. Hirschmann, A.C. Withers, $\mathrm{CO}_{2}$ solubility in Martian basalts and Martian atmospheric evolution. Geochim. Cosmochim. Acta 75, 5987-6003 (2011)

D.E. Smith, M.T. Zuber, H.V. Frey, J.B. Garvin, J.W. Head, D.O. Muhleman, G.H. Pettengill, R.J. Phillips, S.C. Solomon, H.J. Zwally, W.B. Banerdt, T.C. Duxbury, M.P. Golombek, F.G. Lemoine, G.A. Neumann, D.D. Rowlands, O. Aharonson, P.G. Ford, A.B. Ivanov, C.L. Johnson, P.J. McGovern, J.B. Abshire, R.S. Afzal, X. Sun, Mars Orbiter Laser Altimeter: Experiment summary after the first year of global mapping of Mars. J. Geophys. Res. 106, 23689-23722 (2001)

V.V. Shuvalov, N.A. Artemieva, Atmospheric erosion and radiation impulse induced by impacts, in Proc. International Conference on Catastrophic Events and Mass Extinctions: Impacts and Beyond, abs. 3060 (2001)

A. Skumanich, J.A. Eddy, Aspects of long-term variability in Sun and stars, in Solar phenomena in stars and stellar systems, D. Reidel Publishing Co., Dordrecht, 1981, pp. 349-397

R.J. Soare, J.S. Kargel, G.R. Osinski, F. Costard, Thermokarst processes and the origin of crater-rim gullies in Utopia and western Elysium Planitia. Icarus 191, 95-112 (2007)

S.C. Solomon, O. Aharonson, J.M. Aurnou, W.B. Banerdt, M.H. Carr, A.J. Dombard, H.V. Frey, M.P. Golombek, S.A. Hauck II, J.W. Head III, B.M. Jakosky, C.L. Johnson, P.J. McGovern, G.A. Neumann, R.J. Phillips, D.E. Smith, M.T. Zuber, New Perspectives on Ancient Mars. Science, 307, 1214-1220 (2005)

S.C. Solomon, J.W. Head, If the Late Heavy Bombardment of the Moon Was a Terminal Cataclysm, What are Some Implications for Mars? 38 ${ }^{\text {th }}$ LPSC, abs. 1636, p. 1338 (2007)

S.W. Squyres, J.F. Kasting, Early Mars: How warm and how wet? Science 265, 744-749 (1994)

D.I. Steel, Collisions in the Solar System - II. Asteroid impacts upon Mars. Royal Astron. Soc. Month. Not. 215, 369-381 (1985)

R.G. Strom, R. Malhotra, T. Ito, F. Yoshida, D.A. Kring, The origin of planetary impactors in the inner Solar System. Science 309, 1847-1849 (2005)

V.V. Svetsov, Atmospheric erosion and replenishment induced by impacts of cosmic bodies upon the Earth and Mars. Sol. System Res. 41, 28-41 (2007)

K.L. Tanaka, The Stratigraphy of Mars. J. Geophys. Res. 91, 139-158 (1986)

F. Tera, D.A. Papanastassiou, G.J. Wasserburg, Isotopic evidence for a terminal lunar cataclysm. Icarus 68, 1-21 (1974) 
N. Terada, Yu.N. Kulikov, H. Lammer, H.I.M. Lichtenegger, T. Tanaka, H. Shinagawa, T.-L. Zhang, Atmosphere and water loss form early Mars under extreme solar wind and extreme ultraviolet conditions. Astrobiology 9, 55-70, (2009)

C. Thomas, O. Mousis, S. Picaud, V. Ballenegger, Variability of the methane trapping in Martian subsurface clathrate hydrates. Planet. Space Sci. 57, 42-47 (2009)

F. Tian, O.B. Toon, A.A. Pavlov, H. De Sterck, A hydrogen-rich early Earth atmosphere. Science 308, 1014-1017 (2005)

F. Tian, J.F. Kasting, H. Liu, R.G. Roble, Hydrodynamic planetary thermosphere model: 1. The response of the Earths thermosphere to extreme solar EUV conditions and the significance of adiabatic cooling. J. Geophys. Res. 113, Issue E5, CiteID E05008 (2008)

F. Tian, J.F. Kasting, S.C. Solomon, Thermal escape of carbon from the early Martian atmosphere. Geophys. Res. Lett. 36, Issue 2, CiteID L02205 (2009)

T.N. Titus, H.H. Kieffer, P.R. Christensen, Exposed water ice discovered near the south pole of Mars. Science 299, 1048-1051 (2003)

O.B. Toon, T. Segura, K. Zahnle, The formation of martian river valleys by impacts. Ann. Rev. Earth Planet. Sci. 38, 303-322 (2010)

G. Turner, S.F. Knott, R.D. Ash, J.D. Gilmour J.D., Ar-Ar chronology of the martian meteorite ALH 84001: Evidence for the timing of the early bombardment of Mars. Geochim. Cosmochim. Acta 61, 3835-3850 (1997)

A. Valeille, M.R. Combi, V. Tenishev, S.W. Bougher, A.F. Nagy, A study of suprathermal oxygen atoms in Mars upper thermosphere and exosphere over the range of limiting conditions. Icarus 206, 18-27 (2010)

A.M. Vickery, Impacts and atmospheric erosion on the early Earth, in Proc. International workshop on meteorite impact on the early Earth, 51-52 (1990)

Wadhwa, M., 2001. Redox state of Mars: Upper mantle and crust from Eu anomalies in shergottite pyroxenes. Science 291, 1527-1530 (2001)

K.J. Walsh, A. Morbidelli, S.N. Raymond, D.P. O'Brien, A.M. Mandell, A low mass for Mars from Jupiters early gas-driven migration. Nature 475, 206-209 (2011)

J.C.G. Walker, Impact erosion of planetary atmospheres. Icarus 68, 87-98 (1986)

P.H. Warren, G.W. Kallmeyen, Siderophile trace elements in ALH84001, other SNC meteorites and eucrites: evidence of heterogeneity, possibly time-linked, in the mantle of Mars. Meteorit. Planet. Sci. 31, 97-105 (1996)

A.J. Watson, T.M. Donahue, J.C.G. Walker, The dynamics of a rapidly escaping atmosphere: applications to the evolution of Earth and Venus. Icarus 48, 150-166 (1981)

L.L. Watson, L.L Watson, I.D. Hutcheon, S. Epstein, E.M. Stolper, Water on Mars: Clues from deuterium/hydrogen and water contents of hydrous phases in SNC meteorites. Science 265, 86-90 (1994)

H. Wänke, G. Dreibus, Chemistry and accretion history of Mars. Philos. Trans. R. Soc. London 349, 285-293 (1994)

S.C. Werner, Major aspects of the chronostratigraphy and geologic evolutionary history of Mars. PhD thesis, Freien Universitt Berlin, Berlin (2005)

G.W. Wetherill, Late heavy bombardment of the moon and terrestrial planets, in Proc. $6^{\text {th }}$ Lunar Science Conference, (Houston, Texas, Pergamon Press, New York., 1975), pp. 15391561

G.W. Wetherill, Accumulation of terrestrial planets and implications concerning lunar origin, in Origin of the Moon, ed. W.K. Hartmann, R.J. Phillips, G.J. Taylor, Arizona Press, Tucson, 1986, pp 519-550

R.S. Wolff, B.E. Goldstein, C.M. Yeates, The onset and development of Kelvin-Helmholtz instability at the Venus ionopause. J. Geophys. Res. 85, 7697-7707 (1980)

R. Wordsworth, Transient conditions for biogenesis on low-mass exoplanets with escaping hydrogen atmospheres. Icarus 219 267-273 (2012)

Y.L. Yung, J.S. Wen, J.P. Pinto, M. Allen, K.K. Pierce, S. Paulson, HDO in the martian atmosphere: Implications for the abundance of crustal water. Icarus 76, 146-159 (1988)

K.J. Zahnle, J.C.G. Walker, The evolution of solar ultraviolet luminosity. Rev. Geophys. 20, 280-292 (1982)

K.J. Zahnle, J.F. Kasting, Mass fractionation during transonic escape and implications for loss of water from Mars and Venus. Icarus 68, 462-480 (1986)

K.J. Zahnle, J.F. Kasting, J.B. Pollack, Evolution of a steam atmosphere during Earth's accreation. Icarus 74, 62-97 (1988) 
K.J. Zahnle, Xenological constraints on the impact erosion of the early martian atmosphere. J. Geophys. Res. 98, 10899-10913 (1993)

K.J. Zahnle, J.B. Pollack, D. Grinspoon, Impact-generated atmospheres over Titan, Ganymede and Callisto. Icarus 95, 1-23 (1992)

K.J. Zahnle, R.M. Haberle, D.C. Catling, J.F. Kasting, Photochemical instability of the ancient Martian atmosphere. J. Geophys. Res. 113, E11 CiteID E11004 (2008)

K.J. Zahnle, R.S. Freedman, D.C. Catling, Is there methane on Mars? Icarus 212, 493-503 (2011)

A.P. Zent, R.C. Quinn, Simultaneous adsorption of $\mathrm{CO}_{2}$ and $\mathrm{H}_{2} \mathrm{O}$ under Mars-like conditions and application to the evolution of the martian climate. J. Geophys. Res. 100, 5341-5349 (1995) 